\title{
An upper bound for the number of solutions of a system of congruences
}

by

\author{
LYN DodD (Nottingham)
}

1. Introduction. Let $X$ denote an indeterminate. For each vector $\mathbf{m} \in$ $\mathbb{Z}^{2 r}$ with components satisfying $0<m_{i} \leq h$ define

$$
f_{1}(X)=\prod_{i=1}^{r}\left(X+m_{i}\right) \quad \text { and } \quad f_{2}(X)=\prod_{i=r+1}^{2 r}\left(X+m_{i}\right) .
$$

In [1] Burgess showed that, for any prime $p>3$ and primitive character $\chi$ $\left(\bmod p^{\alpha}\right)$, the estimate

$$
\left|\sum_{n=N+1}^{N+H} \chi(n)\right|=O\left(H^{1-1 / r} p^{\alpha(r+1) / 4 r^{2}+\varepsilon}\right)
$$

holds in the case $r=3$. This inequality was obtained by estimating

$$
\sum_{\mathbf{m}}\left|\sum_{x \in A_{1}} \chi\left(\frac{f_{1}}{f_{2}}(x)\right)\right|
$$

where

$$
A_{1}=\left\{x: 0 \leq x<p^{\alpha}, p \nmid f_{1}(x) f_{2}(x)\right\} .
$$

In order to do this, Burgess found estimates for the inner summation over various subsets of $A_{1}$ and then counted the number of $\mathbf{m}$ for which these subsets were non-empty. The counting process was carried out using different methods, one of which concerned the estimation of the cardinality of

$$
S=\left\{\mathbf{m}: 0<m_{i} \leq h, f_{1}(X) \equiv f_{2}(X)\left(\bmod p^{\mu}\right)\right\} .
$$

The estimation of such character sums in the case $r=2$ is contained in [2]. The case $r=4$ has yet to be proved. This paper estimates \#S when $r=4$, as a step in the direction of a proof. The result obtained is given by the following theorem.

The contents of this paper formed part of the author's PhD thesis (Nottingham University, 1991) which was supported by Science and Engineering Research Council. 
TheOREM 1. Suppose $p$ is a prime greater than 5 and $\mu$ is a positive integer. If

$$
H=\left\{\mathbf{m}: 0<m_{i} \leq h \text { for } i=1, \ldots, 8 \text { and } f_{1}(X) \equiv f_{2}(X)\left(\bmod p^{\mu}\right)\right\}
$$

then

$$
\# H \ll \mu^{6}\left(\frac{h^{8}}{p^{3 \mu+[\mu / 2]-[\mu / 4]}}+\frac{h^{6}}{p^{\mu+[\mu / 2]-[\mu / 4]}}+\frac{h^{5}}{p^{\mu-[\mu / 2]}}+h^{4}\right) .
$$

In [4] Hua and Min obtain an asymptotic formula for the number of solutions of the system

$$
x_{1}^{h}+\ldots+x_{s}^{h} \equiv y_{1}^{h}+\ldots+y_{s}^{h}\left(\bmod p^{l}\right) \quad(1 \leq h \leq k)
$$

where $s, k, h, l$ are integers such that $s \geq k \geq 4, l \geq k^{2}$ and $p$ is a prime greater than $k$. Assuming that $p \neq 5$ and letting $s_{r}(\mathbf{x})=\sum_{i=1}^{4} x_{i}^{r}$, in the particular case $s=k=4, l=16$ the number of solutions of the system

$$
\left.\begin{array}{l}
s_{1}(\mathbf{x}) \equiv s_{1}(\mathbf{y}) \\
s_{2}(\mathbf{x}) \equiv s_{2}(\mathbf{y}) \\
s_{3}(\mathbf{x}) \equiv s_{3}(\mathbf{y}) \\
s_{4}(\mathbf{x}) \equiv s_{4}(\mathbf{y})
\end{array}\right\} \quad\left(\bmod p^{16}\right)
$$

is $p^{76}\left(1+O\left(p^{-1 / 4}\right)\right)$. Writing

$$
\begin{aligned}
& \sigma_{1}(\mathbf{x})=x_{1}+x_{2}+x_{3}+x_{4}, \\
& \sigma_{2}(\mathbf{x})=x_{1} x_{2}+x_{1} x_{3}+x_{1} x_{4}+x_{2} x_{3}+x_{2} x_{4}+x_{3} x_{4}, \\
& \sigma_{3}(\mathbf{x})=x_{1} x_{2} x_{3}+x_{1} x_{2} x_{4}+x_{1} x_{3} x_{4}+x_{2} x_{3} x_{4}, \\
& \sigma_{4}(\mathbf{x})=x_{1} x_{2} x_{3} x_{4},
\end{aligned}
$$

it follows that

$$
\begin{aligned}
s_{1}(\mathbf{x})= & \sigma_{1}(\mathbf{x}) \\
s_{2}(\mathbf{x})= & \left(\sigma_{1}(\mathbf{x})\right)^{2}-2 \sigma_{2}(\mathbf{x}), \\
s_{3}(\mathbf{x})= & \left(\sigma_{1}(\mathbf{x})\right)^{3}-3 \sigma_{1}(\mathbf{x}) \sigma_{2}(\mathbf{x})+3 \sigma_{3}(\mathbf{x}), \\
s_{4}(\mathbf{x})= & \left(\sigma_{1}(\mathbf{x})\right)^{4}-4\left(\sigma_{1}(\mathbf{x})\right)^{2} \sigma_{2}(\mathbf{x}) \\
& +4 \sigma_{1}(\mathbf{x}) \sigma_{3}(\mathbf{x})+2\left(\sigma_{2}(\mathbf{x})\right)^{2}-4 \sigma_{4}(\mathbf{x}) .
\end{aligned}
$$

Since $p>5$ the systems (1) and

$$
\left.\begin{array}{l}
\sigma_{1}(\mathbf{x}) \equiv \sigma_{1}(\mathbf{y}) \\
\sigma_{2}(\mathbf{x}) \equiv \sigma_{2}(\mathbf{y}) \\
\sigma_{3}(\mathbf{x}) \equiv \sigma_{3}(\mathbf{y}) \\
\sigma_{4}(\mathbf{x}) \equiv \sigma_{4}(\mathbf{y})
\end{array}\right\} \quad\left(\bmod p^{16}\right)
$$


are equivalent. But (2) holds if and only if

$$
\prod_{i=1}^{4}\left(X+x_{i}\right) \equiv \prod_{i=1}^{4}\left(X+y_{i}\right)\left(\bmod p^{16}\right)
$$

for indeterminate $X$, which, by Theorem 1 , has $\ll p^{76}$ solutions in one complete system of residues. A comparison with the result of Min and Hua shows that, in this case, Theorem 1 is essentially best possible.

2. Basic estimates. The basic tools used in proving Theorem 1 are the well-known estimate in Lemma 2 and Proposition 3 which is reproduced from [3]. The notation $\lceil x\rceil$ denotes the least integer greater than or equal to $x$ and $p^{\alpha} \| x$ means $p^{\alpha} \mid x, p^{\alpha+1} \nmid x$.

Lemma 2. Suppose $p$ is an odd prime and $\nu$ is a positive integer. If $0<x \leq h$ then the number of solutions of the congruence $x^{2}+A x+B \equiv 0$ $\left(\bmod p^{\nu}\right)$ is $\ll h / p^{[(\nu+1) / 2]}+1$.

Proposition 3. Let $f$ be a polynomial of degree $n$ having integer coefficients. Let $p$ be a prime, $d$ be a positive integer, and $\alpha, \beta$ and $\gamma$ be non-negative integers satisfying $\gamma=\lceil\alpha / d\rceil$. If $T=\{x \in$ a complete set of residues $\left.\left(\bmod p^{\gamma}\right): p^{\alpha+\beta} \mid f(x), p^{\beta} \| f^{(d)}(x)\right\}$ then $\# T \ll n$.

If $g(x)$ is a polynomial with integer coefficients such that $p^{\delta} \| g^{(d)}(x)$ then it follows from Proposition 3 that the number of $x$ satisfying $0<x \leq h$ and $g(x) \equiv 0\left(\bmod p^{\mu}\right)$ is $\ll h / p^{\mu-\delta}+1$ if $d=1$ and $\ll h / p^{[(\mu-\delta+1) / 2]}+1$ if $d=2$. The proof of Theorem 1 will be given by a series of lemmas. Throughout we shall use the fact that the conditions $A_{1}+\ldots+A_{n} \equiv 0$ $\left(\bmod p^{\alpha}\right)$ and $p^{a_{j}} \| A_{j}$ for $j=1, \ldots, n$ imply that $a_{k} \geq \min \left(\min _{j \neq k} a_{j}, \alpha\right)$ for $k=1, \ldots, n$.

3. Initial transformations. Making the substitution $M_{i}=m_{i}-m_{1}$ for $i=2, \ldots, 8$ we see that $f_{1}(X) \equiv f_{2}(X)\left(\bmod p^{\mu}\right)$ if and only if the following congruences hold simultaneously:

$$
\begin{gathered}
M_{2} M_{3}+M_{2} M_{4}+M_{3} M_{4} \\
\equiv M_{5} M_{6}+M_{5} M_{7}+M_{5} M_{8}+M_{6} M_{7}+M_{6} M_{8}+M_{7} M_{8}\left(\bmod p^{\mu}\right), \\
M_{2} M_{3} M_{4} \equiv M_{5} M_{6} M_{7}+M_{5} M_{6} M_{8}+M_{5} M_{7} M_{8}+M_{6} M_{7} M_{8}\left(\bmod p^{\mu}\right), \\
0 \equiv M_{5} M_{6} M_{7} M_{8}\left(\bmod p^{\mu}\right) .
\end{gathered}
$$

Eliminating $M_{2}$ from the second and third congruences of the above system produces the pair of congruences

$$
\left(M_{3}+M_{4}\right)\left(M_{5}+M_{6}+M_{7}+M_{8}-M_{4}\right)-M_{3}^{2}
$$




$$
\equiv M_{5} M_{6}+M_{5} M_{7}+M_{5} M_{8}+M_{6} M_{7}+M_{6} M_{8}+M_{7} M_{8}\left(\bmod p^{\mu}\right)
$$

and

$$
\begin{aligned}
& M_{3} M_{4}\left(M_{5}+M_{6}+M_{7}+M_{8}-M_{3}-M_{4}\right) \\
& \quad \equiv M_{5} M_{6} M_{7}+M_{5} M_{6} M_{8}+M_{5} M_{7} M_{8}+M_{6} M_{7} M_{8}\left(\bmod p^{\mu}\right),
\end{aligned}
$$

which together imply that

$$
\begin{array}{r}
\left(M_{4}-M_{8}\right)\left(M_{5} M_{6}+M_{5} M_{7}+M_{6} M_{7}+M_{4}^{2}-M_{4}\left(M_{5}+M_{6}+M_{7}\right)\right) \\
\equiv M_{5} M_{6} M_{7}\left(\bmod p^{\mu}\right) .
\end{array}
$$

Define $\gamma_{5}, \gamma_{6}, \gamma_{7}, \gamma_{8}$ by

$$
\begin{gathered}
p^{\gamma_{5}}\left\|\left(M_{5}, p^{\mu}\right), \quad p^{\gamma_{5}+\gamma_{6}}\right\|\left(M_{5} M_{6}, p^{\mu}\right), \\
p^{\gamma_{5}+\gamma_{6}+\gamma_{7}} \|\left(M_{5} M_{6} M_{7}, p^{\mu}\right), \quad \gamma_{5}+\gamma_{6}+\gamma_{7}+\gamma_{8}=\mu .
\end{gathered}
$$

It may be assumed that $\gamma_{5} \geq \gamma_{6} \geq \gamma_{7} \geq \gamma_{8} \geq 0$, by re-ordering $M_{5}, M_{6}, M_{7}$, $M_{8}$ if necessary, and that for $i=5,6,7$ any power of $p$ dividing $M_{i+1}$ also divides $M_{i}$. Let $\varepsilon, k, m$ be given by

$$
\begin{gathered}
p^{\varepsilon} \|\left(M_{5} M_{6}+M_{5} M_{7}+M_{6} M_{7}+M_{4}^{2}-M_{4}\left(M_{5}+M_{6}+M_{7}\right), p^{\mu}\right), \\
p^{k} \|\left(2 M_{3}+M_{4}-M_{5}-M_{6}-M_{7}, p^{\mu}\right)
\end{gathered}
$$

and

$$
p^{m} \|\left(2 M_{4}-M_{5}-M_{6}-M_{7}, p^{\mu}\right) .
$$

Writing $M_{1}=m_{1}$ it follows that the number of $\mathbf{m}=\left(m_{1}, \ldots, m_{8}\right)$ satisfying $f_{1}(X) \equiv f_{2}(X)\left(\bmod p^{\mu}\right)$ is less than or equal to the number of solutions in $M_{1}, \ldots, M_{8}$ of (3)-(6) with $\left|M_{i}\right|<h$ for $i=1, \ldots, 8$. We now present the lemmas which together provide the proof of Theorem 1 . In all cases there are $h$ possible values for $m_{1}$. Given $M_{3}, \ldots, M_{8}$ there are $\ll h / p^{\mu}+1$ choices for $M_{2}$ from (3). We have $\ll h / p^{\max (\mu-k, k)}+1$ choices for $M_{3}$ from (5) and (9), given $M_{4}, \ldots, M_{8}$. The following notation will be used:

and

$$
A=\frac{h^{8}}{p^{3 \mu+[\mu / 2]-[\mu / 4]}}+\frac{h^{6}}{p^{\mu+[\mu / 2]-[\mu / 4]}}+\frac{h^{5}}{p^{\mu-[\mu / 2]}}+h^{4}
$$

$$
S=\text { " } M_{1}, \ldots, M_{8}:\left|M_{i}\right|<h \text { for } i=1, \ldots, 8 \text { and (3)-(10) hold". }
$$

4. Extending the conditions $S$. In this section we obtain the required estimate except for the set $\{S: 0<m<\mu-[\mu / 4], \varepsilon<\mu,[\mu / 4]<k \leq[\mu / 2]$, $\gamma_{8}>0$ and $\left.p \mid M_{4}\right\}$.

Lemma 4. If $H_{1}=\{S: m=0\}$ then $\# H_{1} \ll \mu^{5} A$.

Proof. Given $M_{4}, M_{5}, M_{6}, M_{7}$ there are $\ll h / p^{\mu-\varepsilon}+1$ choices for $M_{8}$ from (6) and (8). Since $m=0$ there are only non-singular solutions for $M_{4}$ 
from (8) and so we have $\ll h / p^{\varepsilon}+1$ choices for $M_{4}$ given $M_{5}, M_{6}, M_{7}$. By (7) it follows that

$$
\begin{aligned}
\# H_{1} & \ll h\left(\frac{h}{p^{\mu}}+1\right) \sum_{k}\left(\frac{h}{p^{\max (\mu-k, k)}}+1\right) \\
& \times \sum_{\gamma_{5}, \gamma_{6}, \gamma_{7}}\left(\frac{h}{p^{\gamma_{5}}}+1\right)\left(\frac{h}{p^{\gamma_{6}}}+1\right)\left(\frac{h}{p^{\gamma_{7}}}+1\right) \sum_{\varepsilon}\left(\frac{h}{p^{\varepsilon}}+1\right)\left(\frac{h}{p^{\mu-\varepsilon}}+1\right) \\
& \ll \mu^{5} A .
\end{aligned}
$$

Lemma 5. If $H_{2}=\left\{S: m>0\right.$ and $\left.p \nmid M_{4}\right\}$ then $\# H_{2} \ll \mu^{4} A$.

Proof. Given $M_{4}, M_{5}, M_{6}, M_{7}$ there are $\ll h / p^{\mu-\varepsilon}+1$ choices for $M_{8}$ from (6) and (8). By (8) and (10) there are $\ll h / p^{\max (\varepsilon-m, m)}+1$ choices for $M_{4}$ given $M_{5}, M_{6}, M_{7}$. Since $p \nmid M_{4}$, (7) and (10) imply that $p \nmid M_{7}$ and so $\gamma_{7}=0$ and $\gamma_{5}+\gamma_{6}=\mu$. From (10) it can be seen that $2 M_{4}=M_{5}+M_{6}+M_{7}+R p^{m}$ for some $R \in \mathbb{Z}$. Substituting for $M_{4}$ in (8) produces

$$
4\left(M_{5} M_{6}+M_{5} M_{7}+M_{6} M_{7}\right) \equiv\left(M_{5}+M_{6}+M_{7}\right)^{2}\left(\bmod p^{\min (2 m, \varepsilon)}\right),
$$

from which we have $\ll h / p^{\min (m,[(\varepsilon+1) / 2])}+1$ choices for $M_{7}$, given $M_{5}, M_{6}$. Therefore

$$
\begin{aligned}
\# H_{2} \ll & h\left(\frac{h}{p^{\mu}}+1\right) \sum_{k}\left(\frac{h}{p^{\max (\mu-k, k)}}+1\right) \\
& \times \sum_{\varepsilon, m}\left(\frac{h}{p^{\max (\varepsilon-m, m)}}+1\right)\left(\frac{h}{p^{\min (m,[(\varepsilon+1) / 2])}}+1\right) \\
& \times\left(\frac{h}{p^{\mu-\varepsilon}}+1\right) \sum_{\gamma_{5}}\left(\frac{h}{p^{\gamma_{5}}}+1\right)\left(\frac{h}{p^{\mu-\gamma_{5}}}+1\right) \\
\ll & \mu^{4} A .
\end{aligned}
$$

Multiplying (5) by 4 and then rearranging yields

$$
\left(2 M_{3}-\Sigma_{1}+M_{4}\right)^{2} \equiv 4 M_{4}\left(\Sigma_{1}-M_{4}\right)-4 \Sigma_{2}+\left(\Sigma_{1}-M_{4}\right)^{2}\left(\bmod p^{\mu}\right)
$$

where $\Sigma_{1}=M_{5}+M_{6}+M_{7}+M_{8}$ and $\Sigma_{2}=M_{5} M_{6}+M_{5} M_{7}+M_{5} M_{8}+$ $M_{6} M_{7}+M_{6} M_{8}+M_{7} M_{8}$. Hence it follows from (9) that

$$
p^{\min (2 k, \mu)} \mid 4 M_{4}\left(\Sigma_{1}-M_{4}\right)-4 \Sigma_{2}+\left(\Sigma_{1}-M_{4}\right)^{2}
$$

and so

$$
\begin{aligned}
& (11) \quad\left(M_{4}+M_{8}-M_{5}-M_{6}-M_{7}\right)^{2} \\
& \equiv 4\left(M_{5} M_{6}+M_{5} M_{7}+M_{6} M_{7}+M_{4}^{2}-M_{4}\left(M_{5}+M_{6}+M_{7}\right)\right)\left(\bmod p^{\min (2 k, \mu)}\right) .
\end{aligned}
$$


Lemma 6. If $H_{3}=\left\{S: m>0, p \mid M_{4}\right.$ and $\left.\varepsilon=\mu\right\}$ then $\# H_{3} \ll \mu^{3} A$.

Proof. From (6) and (8) we know that $M_{5} M_{6} M_{7} \equiv 0\left(\bmod p^{\mu}\right)$ and so, by (7), $\gamma_{5}+\gamma_{6}+\gamma_{7}=\mu$. Using (8) and (11) we obtain

$$
M_{4}+M_{8}-M_{5}-M_{6}-M_{7} \equiv 0\left(\bmod p^{\min (k,[(\mu+1) / 2])}\right),
$$

from which we have $\ll h / p^{\min (k,[(\mu+1) / 2])}+1$ choices for $M_{8}$ given $M_{4}, M_{5}$, $M_{6}, M_{7}$. There are $\ll h / p^{[(\mu+1) / 2]}+1$ choices for $M_{4}$ from (8), given $M_{5}, M_{6}$, $M_{7}$. Thus

$$
\begin{aligned}
\# H_{3} \ll & h\left(\frac{h}{p^{\mu}}+1\right)\left(\frac{h}{p^{\mu-[\mu / 2]}}+1\right) \\
& \times \sum_{\gamma_{5}, \gamma_{6}}\left(\frac{h}{p^{\gamma_{5}}}+1\right)\left(\frac{h}{p^{\gamma_{6}}}+1\right)\left(\frac{h}{p^{\mu-\gamma_{5}-\gamma_{6}}}+1\right) \\
& \times \sum_{k}\left(\frac{h}{p^{\max (\mu-k, k)}}+1\right)\left(\frac{h}{p^{\min (k,[(\mu+1) / 2])}}+1\right) \\
\ll & \mu^{3} A .
\end{aligned}
$$

Lemma 7. If $H_{4}=\left\{S: k>[\mu / 2], m>0, \varepsilon<\mu\right.$ and $\left.p \mid M_{4}\right\}$ then $\# H_{4} \ll \mu^{5} \mathrm{~A}$.

Proof. As $\varepsilon<\mu$ it follows from (8) that $p^{\varepsilon} \|$ RHS and thus $p^{\varepsilon} \|$ LHS of (11). Hence $\varepsilon$ must be even and $p^{\varepsilon / 2} \| M_{4}+M_{8}-M_{5}-M_{6}-M_{7}$. Together with (11) this gives $\ll h / p^{\mu-\varepsilon / 2}+1$ choices for $M_{8}$ given $M_{4}, M_{5}, M_{6}, M_{7}$. There are $\ll h / p^{\varepsilon / 2}+1$ choices for $M_{4}$ from (8), given $M_{5}, M_{6}, M_{7}$. Therefore

$$
\begin{aligned}
\# H_{4} & \ll h\left(\frac{h}{p^{\mu}}+1\right) \sum_{k}\left(\frac{h}{p^{k}}+1\right) \sum_{\gamma_{5}, \gamma_{6}, \gamma_{7}}\left(\frac{h}{p^{\gamma_{5}}}+1\right)\left(\frac{h}{p^{\gamma_{6}}}+1\right) \\
& \times\left(\frac{h}{p^{\gamma_{7}}}+1\right) \sum_{\varepsilon}\left(\frac{h}{p^{\varepsilon / 2}}+1\right)\left(\frac{h}{p^{\mu-\varepsilon / 2}}+1\right) \\
& \ll \mu^{5} A .
\end{aligned}
$$

Lemma 8. If $H_{5}=\left\{S: 0 \leq k \leq[\mu / 4], m>0, \varepsilon<\mu\right.$ and $\left.p \mid M_{4}\right\}$ then $\# H_{5} \ll \mu^{5} A$.

Proof. There are $\ll h / p^{\mu-\varepsilon}+1$ choices for $M_{8}$ from (6) and (8), given $M_{4}, M_{5}, M_{6}, M_{7}$, and $\ll h / p^{\max (\varepsilon-m, m)}+1$ choices for $M_{4}$ from (8) and (10), given $M_{5}, M_{6}, M_{7}$. As in Lemma 5 we have $\ll h / p^{\min (m,[(\varepsilon+1) / 2])}+1$ choices 
for $M_{7}$, given $M_{5}, M_{6}$, and thus

$$
\begin{aligned}
\# H_{5} \ll & h\left(\frac{h}{p^{\mu}}+1\right) \sum_{k}\left(\frac{h}{p^{\mu-k}}+1\right) \\
& \times \sum_{\varepsilon, m}\left(\frac{h}{p^{\max (\varepsilon-m, m)}}+1\right)\left(\frac{h}{p^{\min (m,[(\varepsilon+1) / 2])}}+1\right) \\
& \times\left(\frac{h}{p^{\mu-\varepsilon}}+1\right) \sum_{\gamma_{5}, \gamma_{6}}\left(\frac{h}{p^{\gamma_{5}}}+1\right)\left(\frac{h}{p^{\gamma_{6}}}+1\right) \\
\ll & \mu^{5} A .
\end{aligned}
$$

LEMMA 9. If $H_{6}=\left\{S:[\mu / 4]<k \leq[\mu / 2], m>0, \varepsilon<\mu, \gamma_{8}=0\right.$ and $\left.p \mid M_{4}\right\}$ then $\# H_{6} \ll \mu^{4} A$.

Pr o of. Given $M_{4}, M_{5}, M_{6}, M_{7}$ we have $\ll h / p^{\max (\mu-\varepsilon, k)}+1$ choices for $M_{8}$ from (6), (8) and (11). From (8) there are $\ll h / p^{[(\varepsilon+1) / 2]}+1$ choices for $M_{4}$ given $M_{5}, M_{6}, M_{7}$. Hence, using ( 7 ),

$$
\begin{aligned}
\# H_{6} & \ll h\left(\frac{h}{p^{\mu}}+1\right) \sum_{\gamma_{5}, \gamma_{6}}\left(\frac{h}{p^{\gamma_{5}}}+1\right)\left(\frac{h}{p^{\gamma_{6}}}+1\right)\left(\frac{h}{p^{\mu-\gamma_{5}-\gamma_{6}}}+1\right) \\
& \times \sum_{\varepsilon, k}\left(\frac{h}{p^{[(\varepsilon+1) / 2]}}+1\right)\left(\frac{h}{p^{\mu-k}}+1\right)\left(\frac{h}{p^{\max (\mu-\varepsilon, k)}}+1\right) \\
& \ll \mu^{4} A .
\end{aligned}
$$

LEMma 10. If $H_{7}=\left\{S:[\mu / 4]<k \leq[\mu / 2], m \geq \mu-[\mu / 4], \varepsilon<\mu, \gamma_{8}>0\right.$ and $\left.p \mid M_{4}\right\}$ then $\# H_{7} \ll \mu^{5} A$.

Proof. Given $M_{5}, M_{6}, M_{7}, M_{8}$ there are $\ll h / p^{m}+1$ choices for $M_{4}$ from (10). By (7) it follows that

$$
\begin{aligned}
\# H_{7} & \ll \\
\qquad & \left(\frac{h}{p^{\mu}}+1\right) \sum_{\gamma_{5}, \gamma_{6}, \gamma_{7}}\left(\frac{h}{p^{\gamma_{5}}}+1\right)\left(\frac{h}{p^{\gamma_{6}}}+1\right)\left(\frac{h}{p^{\gamma_{7}}}+1\right)\left(\frac{h}{p^{\mu-\gamma_{5}-\gamma_{6}-\gamma_{7}}}+1\right) \\
& \times \sum_{k}\left(\frac{h}{p^{\mu-k}}+1\right) \sum_{m}\left(\frac{h}{p^{m}}+1\right) \\
\ll & \mu^{5} A .
\end{aligned}
$$

The conditions $S$ have now been extended as follows:

$S^{\prime}=" S: 0<m<\mu-[\mu / 4], \varepsilon<\mu,[\mu / 4]<k \leq[\mu / 2], \gamma_{8}>0$ and $p \mid M_{4}$ "

This notation will be used in the next section. 
5. Extending the conditions $S^{\prime}$. In all the remaining cases we obtain an expression of the form

$$
\# H_{i} \ll h\left(\frac{h}{p^{\mu}}+1\right) \sum\left(\frac{h^{6}}{p^{D_{a}}}+\frac{h^{5}}{p^{D_{b}}}+\frac{h^{4}}{p^{D_{c}}}+h^{3}\right),
$$

where the sum is over a maximum of six variables. It is sufficient to show that $D_{a} \geq 2 \mu+[\mu / 2]-[\mu / 4], D_{b} \geq \mu+[\mu / 2]-[\mu / 4]$ and $D_{c} \geq \mu-[\mu / 2]$ since

$$
h\left(\frac{h}{p^{\mu}}+1\right)\left(\frac{h^{6}}{p^{2 \mu+[\mu / 2]-[\mu / 4]}}+\frac{h^{5}}{p^{\mu+[\mu / 2]-[\mu / 4]}}+\frac{h^{4}}{p^{\mu-[\mu / 2]}}+h^{3}\right) \ll A .
$$

We now continue with further steps in the proof of Theorem 1.

Lemma 11. If $H_{8}=\left\{S^{\prime}: 2 k \leq \varepsilon\right\}$ then $\# H_{8} \ll \mu^{6} A$.

Proof. From (8) and (11) we know that $p^{k} \mid M_{4}+M_{8}-M_{5}-M_{6}-M_{7}$. By (10), this implies that $p^{\min (m, k)} \mid M_{4}-M_{8}$. Hence, by (6) and (7), it follows that

$$
\varepsilon+\min (m, k) \leq \gamma_{5}+\gamma_{6}+\gamma_{7}<\mu .
$$

There are $\ll h / p^{\mu-\varepsilon}+1$ choices for $M_{8}$ from (6) and (8), given $M_{4}, M_{5}, M_{6}$, $M_{7}$, and $\ll h / p^{\max (\varepsilon-m, m)}+1$ choices for $M_{4}$ from (8) and (10), given $M_{5}$, $M_{6}, M_{7}$. Therefore

$$
\begin{aligned}
\# H_{8} \ll & h\left(\frac{h}{p^{\mu}}+1\right) \sum_{k, \varepsilon, m}\left(\frac{h}{p^{\mu-k}}+1\right)\left(\frac{h}{p^{\mu-\varepsilon}}+1\right)\left(\frac{h}{p^{\max (\varepsilon-m, m)}}+1\right) \\
& \times \sum_{\gamma_{5}, \gamma_{6}, \gamma_{7}}\left(\frac{h}{p^{\gamma_{5}}}+1\right)\left(\frac{h}{p^{\gamma_{6}}}+1\right)\left(\frac{h}{p^{\gamma_{7}}}+1\right) \\
\ll & h\left(\frac{h}{p^{\mu}}+1\right) \sum_{\substack{k, \varepsilon, m \\
\gamma_{5}, \gamma_{6}, \gamma_{7}}}\left(\frac{h^{6}}{p^{D_{1}}}+\frac{h^{5}}{p^{D_{2}}}+\frac{h^{4}}{p^{D_{3}}}+h^{3}\right),
\end{aligned}
$$

where

$$
\begin{array}{r}
D_{1}=2 \mu-k-\varepsilon+\max (\varepsilon-m, m)+\gamma_{5}+\gamma_{6}+\gamma_{7}, \\
D_{2}=\min \left(2 \mu-k-\varepsilon+\max (\varepsilon-m, m), 2 \mu-k-\varepsilon+\gamma_{5}+\gamma_{6}+\gamma_{7},\right. \\
\left.\mu-\varepsilon+\max (\varepsilon-m, m)+\gamma_{5}+\gamma_{6}+\gamma_{7}\right), \\
D_{3}=\min \left(\mu-\varepsilon+\max (\varepsilon-m, m), \mu-\varepsilon+\gamma_{5}+\gamma_{6}+\gamma_{7}, 2 \mu-k-\varepsilon,\right. \\
\left.\gamma_{5}+\gamma_{6}+\gamma_{7}+\max (\varepsilon-m, m)\right) .
\end{array}
$$

It can be seen from (12) that $D_{1} \geq 2 \mu-k+\max (\varepsilon-m, m)+\min (m, k) \geq$ $2 \mu+[\mu / 2]-[\mu / 4]$. By (7) we know that $\gamma_{5}+\gamma_{6}+\gamma_{7} \geq \mu-[\mu / 4]>\mu-k$. Also, if $\max (\varepsilon-m, m)>\gamma_{5}+\gamma_{6}+\gamma_{7}$ then $\varepsilon-m>\gamma_{5}+\gamma_{6}+\gamma_{7}$. This is possible only if $m<[\mu / 4]$, in which case (12) implies that $\varepsilon+m \leq \gamma_{5}+\gamma_{6}+\gamma_{7}<\varepsilon-m$, 
a contradiction. We conclude that $\max (\varepsilon-m, m) \leq \gamma_{5}+\gamma_{6}+\gamma_{7}$. Hence $D_{3}=\min (\mu-\varepsilon+\max (\varepsilon-m, m), 2 \mu-k-\varepsilon) \geq \mu-[\varepsilon / 2] \geq \mu-[\mu / 2]$ and $D_{2}=2 \mu-k-\varepsilon+\max (\varepsilon-m, m)$. If $\varepsilon>[\mu / 2]+[\mu / 4]$ then (12) implies that $m \leq[\mu / 4]$ and so $D_{2}=2 \mu-k-m \geq 2 \mu-[\mu / 2]-[\mu / 4]$. If $\varepsilon \leq[\mu / 2]+[\mu / 4]$ then, as $k \leq \max (\varepsilon-m, m)$, we have $D_{2} \geq 2 \mu-\varepsilon \geq 2 \mu-[\mu / 2]-[\mu / 4]$.

It may now be assumed that $\mu \geq 2 k>\varepsilon$. Consequently, it follows from (8) and (11) that $\varepsilon$ is even and

$$
p^{\varepsilon / 2} \| M_{4}+M_{8}-M_{5}-M_{6}-M_{7} .
$$

Denote by $Q$ the expression

$$
Q=M_{5} M_{6}+M_{5} M_{7}+M_{6} M_{7}+M_{4}^{2}-M_{4}\left(M_{5}+M_{6}+M_{7}\right) .
$$

Lemma 12. If $H_{9}=\left\{S^{\prime}: 2 k>\varepsilon, 2 m>\varepsilon\right\}$ then $\# H_{9} \ll \mu^{5} A$.

Proof. Given $M_{4}, M_{5}, M_{6}, M_{7}$ there are $\ll h / p^{\max (\mu-\varepsilon, 2 k-\varepsilon / 2)}+1$ choices for $M_{8}$ from (6), (8), (11) and (13). By (10), $p^{2 m} \|\left(2 M_{4}-M_{5}-\right.$ $\left.M_{6}-M_{7}\right)^{2}$ and so

$$
4 Q \equiv 2\left(M_{5} M_{6}+M_{5} M_{7}+M_{6} M_{7}\right)-M_{5}^{2}-M_{6}^{2}-M_{7}^{2}\left(\bmod p^{2 m}\right) .
$$

As $p^{\varepsilon} \| Q$ we deduce that

$$
p^{\varepsilon} \| M_{5}^{2}+M_{6}^{2}+M_{7}^{2}-2\left(M_{5} M_{6}+M_{5} M_{7}+M_{6} M_{7}\right) .
$$

It follows from (10) and (13) that $p^{\varepsilon / 2} \mid M_{4}-M_{8}$, which, together with (6) and (7), implies that

$$
3 \varepsilon / 2 \leq \gamma_{5}+\gamma_{6}+\gamma_{7}<\mu .
$$

Thus from (6) we obtain

$$
\frac{Q}{p^{\varepsilon}} \cdot \frac{M_{4}-M_{8}}{p^{\varepsilon / 2}} \equiv \frac{M_{5} M_{6} M_{7}}{p^{3 \varepsilon / 2}}\left(\bmod p^{\mu-3 \varepsilon / 2}\right)
$$

and from (11) and (13) we have

$$
\left(\frac{Q}{p^{\varepsilon}} \cdot \frac{M_{4}+M_{8}-M_{5}-M_{6}-M_{7}}{p^{\varepsilon / 2}}\right)^{2} \equiv \frac{4 Q^{3}}{p^{3 \varepsilon}}\left(\bmod p^{2 k-\varepsilon}\right) .
$$

Since $M_{4}+M_{8}-M_{5}-M_{6}-M_{7}=2 M_{4}-M_{5}-M_{6}-M_{7}-\left(M_{4}-M_{8}\right)$, combining the above two congruences produces

$$
\left(\frac{Q\left(2 M_{4}-M_{5}-M_{6}-M_{7}\right)-M_{5} M_{6} M_{7}}{p^{3 \varepsilon / 2}}\right)^{2} \equiv \frac{4 Q^{3}}{p^{3 \varepsilon}}\left(\bmod p^{\min (\mu-3 \varepsilon / 2,2 k-\varepsilon)}\right),
$$

which simplifies to

$$
Q^{2}\left(M_{5}^{2}+M_{6}^{2}+M_{7}^{2}-2\left(M_{5} M_{6}+M_{5} M_{7}+M_{6} M_{7}\right)\right)
$$

$\equiv M_{5} M_{6} M_{7}\left(2 Q\left(2 M_{4}-M_{5}-M_{6}-M_{7}\right)-M_{5} M_{6} M_{7}\right)\left(\bmod p^{\min (\mu+3 \varepsilon / 2,2 k+2 \varepsilon)}\right)$. 
From (15) it can be seen that $p^{3 \varepsilon} \|$ LHS of (17). By (16) we must have $\min (\mu+3 \varepsilon / 2,2 k+2 \varepsilon)>3 \varepsilon$ and thus $p^{3 \varepsilon} \| \operatorname{RHS}$ of $(17)$, or

$$
p^{3 \varepsilon-\gamma_{5}-\gamma_{6}-\gamma_{7}} \| 2 Q\left(2 M_{4}-M_{5}-M_{6}-M_{7}\right)-M_{5} M_{6} M_{7} .
$$

This together with (10) implies that

$$
3 \varepsilon-\gamma_{5}-\gamma_{6}-\gamma_{7} \geq \min \left(\varepsilon+m, \gamma_{5}+\gamma_{6}+\gamma_{7}\right) .
$$

If $\varepsilon+m \leq \gamma_{5}+\gamma_{6}+\gamma_{7}$ then $2 \varepsilon-m \geq \gamma_{5}+\gamma_{6}+\gamma_{7} \geq \varepsilon+m$, contradicting $2 m>\varepsilon$. Hence, from (7), (16) and the above we conclude that

$$
\mu-\left[\frac{\mu}{4}\right] \leq \frac{3 \varepsilon}{2}=\gamma_{5}+\gamma_{6}+\gamma_{7}<\mu .
$$

It follows from (8), (10) and (18) that

$$
\begin{array}{r}
p^{5 \varepsilon / 2} \| 2 Q\left(2 M_{4}-M_{5}-M_{6}-M_{7}\right)\left(M_{5}^{2}+M_{6}^{2}+M_{7}^{2}-2\left(M_{5} M_{6}+M_{5} M_{7}+M_{6} M_{7}\right)\right) \\
-2 M_{5} M_{6} M_{7}\left(2 Q+\left(2 M_{4}-M_{5}-M_{6}-M_{7}\right)^{2}\right) .
\end{array}
$$

This is the derivative of (17) with respect to $M_{4}$ and so there are « $h / p^{\min (\mu-\varepsilon, 2 k-\varepsilon / 2)}+1$ choices for $M_{4}$ given $M_{5}, M_{6}, M_{7}$. By (7) and (18) it follows that

$$
\begin{aligned}
& \# H_{9} \ll \\
& \quad \quad\left(\frac{h}{p^{\mu}}+1\right) \sum_{k, \varepsilon}\left(\frac{h}{p^{\min (\mu-\varepsilon, 2 k-\varepsilon / 2)}}+1\right)\left(\frac{h}{p^{\max (\mu-\varepsilon, 2 k-\varepsilon / 2)}}+1\right) \\
& \sum_{\gamma_{5}, \gamma_{6}, \gamma_{7}}\left(\frac{h}{p^{\gamma_{5}}}+1\right)\left(\frac{h}{p^{\gamma_{6}}}+1\right)\left(\frac{h}{p^{\gamma_{7}}}+1\right) \\
& \ll \mu^{3} h\left(\frac{h}{p^{\mu}}+1\right) \sum_{k, \varepsilon}\left(\frac{h^{6}}{p^{D_{4}}}+\frac{h^{5}}{p^{D_{5}}}+\frac{h^{4}}{p^{D^{6}}}+h^{3}\right),
\end{aligned}
$$

where

$$
\begin{aligned}
D_{4} & =2 \mu+k \geq 2 \mu+\left[\frac{\mu}{2}\right]-\left[\frac{\mu}{4}\right], \\
D_{5} & =\min \left(2 \mu+k-\frac{3 \varepsilon}{2}, 2 \mu-k+\frac{\varepsilon}{2}, \mu+k+\varepsilon\right) \\
& >\min (2 \mu-\varepsilon, \mu+k) \geq \mu+\left[\frac{\mu}{2}\right]-\left[\frac{\mu}{4}\right], \\
D_{6} & =\min \left(\mu+2 k-\frac{3 \varepsilon}{2}, 2 \mu-k-\varepsilon, \mu+k-\frac{\varepsilon}{2}, \mu-k+\frac{3 \varepsilon}{2}\right) \\
& >\mu-k \geq \mu-\left[\frac{\mu}{2}\right] .
\end{aligned}
$$

Lemma 13. If $H_{10}=\left\{S^{\prime}: 2 k>\varepsilon>2 m\right\}$ then $\# H_{10} \ll \mu^{6} A$. 
Proof. Given $M_{4}, M_{5}, M_{6}, M_{7}$ there are $\ll h / p^{\max (\mu-\varepsilon, 2 k-\varepsilon / 2)}+1$ choices for $M_{8}$ from (6), (8), (11) and (13). Since $p \neq 2$, from (8) and (14) we know that $p^{\varepsilon} \| 4 Q$. This can be rewritten as

$$
\begin{aligned}
\left(2 M_{4}-M_{5}-\right. & \left.M_{6}-M_{7}\right)^{2} \\
& \equiv M_{5}^{2}+M_{6}^{2}+M_{7}^{2}-2\left(M_{5} M_{6}+M_{5} M_{7}+M_{6} M_{7}\right)\left(\bmod p^{\varepsilon}\right),
\end{aligned}
$$

which, together with (10), implies that

$$
p^{2 m} \| M_{5}^{2}+M_{6}^{2}+M_{7}^{2}-2\left(M_{5} M_{6}+M_{5} M_{7}+M_{6} M_{7}\right) .
$$

From (10) and (13) we see that $p^{m} \mid M_{4}-M_{8}$ and so from (6) and (7) we have

$$
\varepsilon+m \leq \gamma_{5}+\gamma_{6}+\gamma_{7}<\mu .
$$

Using (6), (11), (13) and (20) we deduce that

$$
\frac{Q}{p^{\varepsilon}} \cdot \frac{M_{4}-M_{8}}{p^{m}} \equiv \frac{M_{5} M_{6} M_{7}}{p^{\varepsilon+m}}\left(\bmod p^{\mu-\varepsilon-m}\right)
$$

and

$$
\left(\frac{Q}{p^{\varepsilon}} \cdot \frac{M_{4}+M_{8}-M_{5}-M_{6}-M_{7}}{p^{m}}\right)^{2} \equiv \frac{4 Q^{3}}{p^{2 \varepsilon+2 m}}\left(\bmod p^{2 k-2 m}\right) .
$$

Combining these two congruences as in the previous lemma, we obtain

$$
Q^{2}\left(M_{5}^{2}+M_{6}^{2}+M_{7}^{2}-2\left(M_{5} M_{6}+M_{5} M_{7}+M_{6} M_{7}\right)\right)
$$

$\equiv M_{5} M_{6} M_{7}\left(2 Q\left(2 M_{4}-M_{5}-M_{6}-M_{7}\right)-M_{5} M_{6} M_{7}\right)\left(\bmod p^{\min (\mu+\varepsilon+m, 2 k+2 \varepsilon)}\right)$.

By (8) and (19), $p^{2 \varepsilon+2 m} \|$ LHS of (21). But from (20) we know that $\min (\mu+$ $\varepsilon+m, 2 k+2 \varepsilon)>2 \varepsilon+2 m$ and so $p^{2 \varepsilon+2 m} \|$ RHS of (21), or

$$
p^{2 \varepsilon+2 m-\gamma_{5}-\gamma_{6}-\gamma_{7}} \| 2 Q\left(2 M_{4}-M_{5}-M_{6}-M_{7}\right)-M_{5} M_{6} M_{7} .
$$

Hence, by (10), we see that $2 \varepsilon+2 m-\gamma_{5}-\gamma_{6}-\gamma_{7} \geq \min \left(\varepsilon+m, \gamma_{5}+\gamma_{6}+\gamma_{7}\right)$, which, together with (7) and (20), implies that

$$
\mu-\left[\frac{\mu}{4}\right] \leq \varepsilon+m=\gamma_{5}+\gamma_{6}+\gamma_{7}<\mu .
$$

Also, from (10), (19) and (22) it can be seen that

$$
\begin{aligned}
p^{4 m} & \|\left(2\left(2 M_{4}-M_{5}-M_{6}-M_{7}\right)^{2}+4 Q\right)\left(M_{5}^{2}+M_{6}^{2}+M_{7}^{2}\right. \\
& \left.-2\left(M_{5} M_{6}+M_{5} M_{7}+M_{6} M_{7}\right)\right)-12 M_{5} M_{6} M_{7}\left(2 M_{4}-M_{5}-M_{6}-M_{7}\right) .
\end{aligned}
$$

This expression is the second derivative of (21) with respect to $M_{4}$ and so there are $\ll h / p^{[(\min (\mu+\varepsilon-3 m, 2 k+2 \varepsilon-4 m)+1) / 2]}+1$ choices for $M_{4}$, given 
$M_{5}, M_{6}, M_{7}$. Hence, by (22),

$$
\begin{aligned}
\# H_{10} \ll & h\left(\frac{h}{p^{\mu}}+1\right) \\
& \times \sum_{k, \varepsilon, m}\left(\frac{h}{p^{\max (\mu-\varepsilon, 2 k-\varepsilon / 2)}}+1\right)\left(\frac{h}{\left.p^{\min ([(\mu+\varepsilon-3 m+1) / 2], k+\varepsilon-2 m)}+1\right)}\right. \\
& \times\left(\frac{h}{p^{\mu-k}}+1\right) \sum_{\gamma_{5}, \gamma_{6}, \gamma_{7}}\left(\frac{h}{p^{\gamma_{5}}}+1\right)\left(\frac{h}{p^{\gamma_{6}}}+1\right)\left(\frac{h}{p^{\gamma_{7}}}+1\right) \\
\ll & \mu^{3} h\left(\frac{h}{p^{\mu}}+1\right) \sum_{k, \varepsilon, m}\left(\frac{h^{6}}{p^{D_{7}}}+\frac{h^{5}}{p^{D_{8}}}+\frac{h^{4}}{p^{D_{9}}}+h^{3}\right),
\end{aligned}
$$

where

$$
\begin{gathered}
D_{7}=\max \left(2 \mu-k, \mu+k+\frac{\varepsilon}{2}\right)+\min \left(\left[\frac{\mu+\varepsilon-m+1}{2}\right], k+\varepsilon-m\right) \\
\geq 2 \mu+\frac{\varepsilon}{2} \geq 2 \mu+\left[\frac{\mu}{2}\right]-\left[\frac{\mu}{4}\right], \\
D_{8}=\mu-k+\min \left(\max \left(\mu-\frac{\varepsilon}{2}, 2 k\right)+\min \left(\left[\frac{\mu-3 m+1}{2}\right], k+\frac{\varepsilon}{2}-2 m\right),\right. \\
\max \left(\mu+m, 2 k+\frac{\varepsilon}{2}+m\right), \\
>\mu+k \geq \mu+\left[\frac{\mu}{2}\right]-\left[\frac{\mu}{4}\right], \\
D_{9}=\min \left(\mu-k+\min \left(\varepsilon+m, \max \left(\mu-\varepsilon, 2 k-\frac{\varepsilon}{2}\right),\right.\right. \\
\left.\min \left(\left[\frac{\mu-3 m+1}{2}\right], k+m\right)\right) \\
\left.>\mu-k \geq \mu-\left[\frac{\mu}{2}\right] \cdot \min \left(\left[\frac{\mu+\varepsilon-3 m+1}{2}\right], k+\varepsilon-2 m\right)\right),
\end{gathered}
$$

It may now be assumed that $\varepsilon=2 m$. There are $\ll h / p^{\max (\mu-2 m, 2 k-m)}+1$ choices for $M_{8}$ from (6), (8), (11) and (13) given $M_{4}, M_{5}, M_{6}, M_{7}$.

Lemma 14. If $H_{11}=\left\{S^{\prime}: 2 k>\varepsilon=2 m\right.$ and $\left.\gamma_{5}+\gamma_{6}+\gamma_{7} \geq 2 k+m\right\}$ then $\# H_{11} \ll \mu^{5} A$. 
Pr o of. From (10) there are $\ll h / p^{m}+1$ choices for $M_{4}$ given $M_{5}, M_{6}, M_{7}$ and it follows that

$$
\begin{aligned}
\# H_{11} \ll & h\left(\frac{h}{p^{\mu}}+1\right) \sum_{k, m}\left(\frac{h}{p^{\max (\mu-2 m, 2 k-m)}}+1\right)\left(\frac{h}{p^{\mu-k}}+1\right)\left(\frac{h}{p^{m}}+1\right) \\
& \times \sum_{\gamma_{5}, \gamma_{6}, \gamma_{7}}\left(\frac{h}{p^{\gamma_{5}}}+1\right)\left(\frac{h}{p^{\gamma_{6}}}+1\right)\left(\frac{h}{p^{\gamma_{7}}}+1\right) \\
\ll & \mu^{3} h\left(\frac{h}{p^{\mu}}+1\right) \sum_{k, m}\left(\frac{h^{6}}{p^{D_{10}}}+\frac{h^{5}}{p^{D_{11}}}+\frac{h^{4}}{p^{D_{12}}}+h^{3}\right),
\end{aligned}
$$

where

$$
\begin{aligned}
D_{10} & =\mu+k+2 m+\max (\mu-2 m, 2 k-m) \geq 2 \mu+k \geq 2 \mu+\left[\frac{\mu}{2}\right]-\left[\frac{\mu}{4}\right], \\
D_{11} & =\min (\mu+k+2 m, \min (\mu-k+m, 2 k+2 m)+\max (\mu-2 m, 2 k-m)) \\
& \geq \mu+k \geq \mu+\left[\frac{\mu}{2}\right]-\left[\frac{\mu}{4}\right], \\
D_{12} & =\min (\max (\mu-m, 2 k), \mu-k+m, 2 k+2 m) \\
& \geq \min (\mu-k, 2 k) \geq \mu-\left[\frac{\mu}{2}\right] .
\end{aligned}
$$

By (10) and (13) we know that $p^{m} \mid M_{4}-M_{8}$ and so, by (6), (7) and (8),

$$
3 m \leq \gamma_{5}+\gamma_{6}+\gamma_{7}<\mu .
$$

Also, with $Q$ given by (14), from (6), (11) and (13) we obtain

$$
\frac{Q}{p^{2 m}} \cdot \frac{M_{4}-M_{8}}{p^{m}} \equiv \frac{M_{5} M_{6} M_{7}}{p^{3 m}}\left(\bmod p^{\mu-3 m}\right)
$$

and

$$
\left(\frac{Q}{p^{2 m}} \cdot \frac{M_{4}+M_{8}-M_{5}-M_{6}-M_{7}}{p^{m}}\right)^{2} \equiv \frac{4 Q^{3}}{p^{6 m}}\left(\bmod p^{2 k-2 m}\right) .
$$

Proceeding as in Lemma 12, these two congruences combine to produce

$$
Q^{2}\left(M_{5}^{2}+M_{6}^{2}+M_{7}^{2}-2\left(M_{5} M_{6}+M_{5} M_{7}+M_{6} M_{7}\right)\right)
$$

$\equiv M_{5} M_{6} M_{7}\left(2 Q\left(2 M_{4}-M_{5}-M_{6}-M_{7}\right)-M_{5} M_{6} M_{7}\right)\left(\bmod p^{\min (2 k+4 m, \mu+3 m)}\right)$.

Define $x$ by

$$
p^{x} \|\left(M_{5}^{2}+M_{6}^{2}+M_{7}^{2}-2\left(M_{5} M_{6}+M_{5} M_{7}+M_{6} M_{7}\right), p^{\mu}\right) .
$$

Lemma 15. If $H_{12}=\left\{S^{\prime}:\right.$ (25) holds, $x \geq 2 k>\varepsilon=2 m$ and $2 k+m>$ $\left.\gamma_{5}+\gamma_{6}+\gamma_{7}\right\}$ then $\# H_{12} \ll \mu^{5} A$. 
Proof. Since $p^{2 m} \| Q(25)$ implies that $p^{\min (2 k+4 m, \mu+3 m)} \mid$ RHS of $(24)$ or, by (7),

$4 Q\left(2 M_{4}-M_{5}-M_{6}-M_{7}\right) \equiv 2 M_{5} M_{6} M_{7}\left(\bmod p^{\min (2 k+4 m, \mu+3 m)-\gamma_{5}-\gamma_{6}-\gamma_{7}}\right)$.

We know that $\min (2 k+4 m, \mu+3 m)-\gamma_{5}-\gamma_{6}-\gamma_{7}>3 m$. Since $p^{3 m} \|$ LHS and $p^{\gamma_{5}+\gamma_{6}+\gamma_{7}} \|$ RHS of the above we conclude that

$$
\mu-\left[\frac{\mu}{4}\right] \leq 3 m=\gamma_{5}+\gamma_{6}+\gamma_{7}<\mu .
$$

As $4 Q=\left(2 M_{4}-M_{5}-M_{6}-M_{7}\right)^{2}-M_{5}^{2}-M_{6}^{2}-M_{7}^{2}+2\left(M_{5} M_{6}+M_{5} M_{7}+M_{6} M_{7}\right)$ we can rewrite the above as

$$
\begin{aligned}
\left(2 M_{4}-M_{5}-\right. & \left.M_{6}-M_{7}\right)\left(\left(2 M_{4}-M_{5}-M_{6}-M_{7}\right)^{2}\right. \\
& \left.\quad-M_{5}^{2}-M_{6}^{2}-M_{7}^{2}+2\left(M_{5} M_{6}+M_{5} M_{7}+M_{6} M_{7}\right)\right) \\
\equiv & 2 M_{5} M_{6} M_{7}\left(\bmod p^{\min (2 k+m, \mu)}\right) .
\end{aligned}
$$

By (10) and (25) it follows that

$$
\begin{aligned}
p^{2 m} \| 6\left(2 M_{4}-\right. & \left.M_{5}-M_{6}-M_{7}\right)^{2} \\
& -2\left(M_{5}^{2}+M_{6}^{2}+M_{7}^{2}-2\left(M_{5} M_{6}+M_{5} M_{7}+M_{6} M_{7}\right)\right) .
\end{aligned}
$$

This is the derivative of the above expression with respect to $M_{4}$ and so there are $\ll h / p^{\min (2 k-m, \mu-2 m)}+1$ choices for $M_{4}$ given $M_{5}, M_{6}, M_{7}$. Therefore

$$
\begin{aligned}
\# H_{12} \ll & h\left(\frac{h}{p^{\mu}}+1\right) \sum_{k, m}\left(\frac{h}{p^{\min (2 k-m, \mu-2 m)}}+1\right) \\
& \times\left(\frac{h}{p^{\max (2 k-m, \mu-2 m)}}+1\right)\left(\frac{h}{p^{\mu-k}}+1\right) \\
& \times \sum_{\gamma_{5}, \gamma_{6}, \gamma_{7}}\left(\frac{h}{p^{\gamma_{5}}}+1\right)\left(\frac{h}{p^{\gamma_{6}}}+1\right)\left(\frac{h}{p^{\gamma_{7}}}+1\right) .
\end{aligned}
$$

As (26) holds, the result follows by comparison with Lemma 12.

Lemma 16. If $H_{13}=\left\{S^{\prime}:(25)\right.$ holds, $2 k>x \geq \mu-m, 2 k>\varepsilon=2 m$ and $\left.2 k+m>\gamma_{5}+\gamma_{6}+\gamma_{7}\right\}$ then $\# H_{13} \ll \mu^{5} A$.

Pr o o f. There are $\ll h / p^{m}+1$ choices for $M_{4}$ from (10) given $M_{5}, M_{6}, M_{7}$ and $\ll h / p^{[(x+1) / 2]}+1$ choices for $M_{7}$ from (25) given $M_{5}, M_{6}$. By (7) and (23),

$$
\begin{aligned}
\# H_{13} \ll & h\left(\frac{h}{p^{\mu}}+1\right) \\
& \times \sum_{k, m, x}\left(\frac{h}{p^{\mu-k}}+1\right)\left(\frac{h}{p^{m}}+1\right)\left(\frac{h}{p^{2 k-m}}+1\right)\left(\frac{h}{p^{[(x+1) / 2]}}+1\right)
\end{aligned}
$$




$$
\begin{aligned}
& \times \sum_{\gamma_{5}, \gamma_{6}}\left(\frac{h}{p^{\gamma_{5}}}+1\right)\left(\frac{h}{p^{\gamma_{6}}}+1\right) \\
& \ll \mu h\left(\frac{h}{p^{\mu}}+1\right) \sum_{\substack{k, m \\
\gamma_{5}, \gamma_{6}}}\left(\frac{h^{6}}{p^{D_{13}}}+\frac{h^{5}}{p^{D_{14}}}+\frac{h^{4}}{p^{D_{15}}}+h^{3}\right),
\end{aligned}
$$

where

$$
\begin{aligned}
D_{13} & =\mu+k+\gamma_{5}+\gamma_{6}+\left[\frac{\mu-m+1}{2}\right] \\
& \geq \mu+2\left[\frac{\mu-m+1}{2}\right]+\max \left(2 m, \mu-\left[\frac{\mu}{2}\right]\right), \\
D_{14} & =\left[\frac{\mu-m+1}{2}\right]+\min \left(\mu+k, \gamma_{5}+\gamma_{6}+\min (\mu-k+m, 2 k)\right) \\
& \geq \mu+\left[\frac{\mu-m+1}{2}\right]>\mu+\left[\frac{\mu}{2}\right]-\left[\frac{\mu}{4}\right], \\
D_{15} & =\min \left(\mu+k,\left[\frac{\mu-m+1}{2}\right]+\min \left(2 k, \gamma_{5}+\gamma_{6}+m, \mu-k+m\right)\right) \\
& >\mu-\left[\frac{\mu}{2}\right] .
\end{aligned}
$$

If $m>[\mu / 4]$ then $D_{13} \geq 2 \mu+m \geq 2 \mu+[\mu / 2]-[\mu / 4]$ and if $m \leq[\mu / 4]$ then $D_{13} \geq 3 \mu-m-[\mu / 2] \geq 3 \mu-[\mu / 2]-[\mu / 4]$.

It remains to consider $\min (2 k, \mu-m)>x$. From (8), (14) and (25) we see that $p^{x+4 m} \|$ LHS of (24) and thus

$$
p^{x+4 m} \| M_{5} M_{6} M_{7}\left(2 Q\left(2 M_{4}-M_{5}-M_{6}-M_{7}\right)-M_{5} M_{6} M_{7}\right) .
$$

This together with (23) implies that $x+4 m \geq \gamma_{5}+\gamma_{6}+\gamma_{7}+3 m$, or

$$
x \geq \gamma_{5}+\gamma_{6}+\gamma_{7}-m .
$$

We now look at the derivatives of (24) with respect to $M_{4}$. Define $T, U$ and $V$ by

$$
\begin{aligned}
& p^{T} \|\left(Q\left(2 M_{4}-M_{5}-M_{6}-M_{7}\right)\right. \\
& \times\left(M_{5}^{2}+M_{6}^{2}+M_{7}^{2}-2\left(M_{5} M_{6}+M_{5} M_{7}+M_{6} M_{7}\right)\right) \\
&-\left.M_{5} M_{6} M_{7}\left(2 Q+\left(2 M_{4}-M_{5}-M_{6}-M_{7}\right)^{2}\right), p^{\min (2 k+4 m, \mu+3 m)}\right), \\
& p^{U} \|\left(\left(2 Q+\left(2 M_{4}-M_{5}-M_{6}-M_{7}\right)^{2}\right)\right. \\
& \quad \times\left(M_{5}^{2}+M_{6}^{2}+M_{7}^{2}-2\left(M_{5} M_{6}+M_{5} M_{7}+M_{6} M_{7}\right)\right) \\
&\left.\quad-6 M_{5} M_{6} M_{7}\left(2 M_{4}-M_{5}-M_{6}-M_{7}\right), p^{\min (2 k+4 m, \mu+3 m)}\right)
\end{aligned}
$$


and

(30) $\quad p^{V} \|\left(\left(2 M_{4}-M_{5}-M_{6}-M_{7}\right)\right.$

$$
\begin{aligned}
& \times\left(M_{5}^{2}+M_{6}^{2}+M_{7}^{2}-2\left(M_{5} M_{6}+M_{5} M_{7}+M_{6} M_{7}\right)\right) \\
& \left.-2 M_{5} M_{6} M_{7}, p^{\min (2 k+4 m, \mu+3 m)}\right)
\end{aligned}
$$

By (7), (10), (25) and (27) it can be seen that

$$
T \geq \gamma_{5}+\gamma_{6}+\gamma_{7}+2 m, \quad U \geq \gamma_{5}+\gamma_{6}+\gamma_{7}+m,
$$

$$
V \geq \gamma_{5}+\gamma_{6}+\gamma_{7}
$$

The conditions $S^{\prime}$ have now been extended. In the final section the following notation will be used:

$$
\begin{aligned}
S^{\prime \prime}=\text { " } & S^{\prime}:(25),(28),(29),(30) \text { hold, } \\
& \min (2 k, \mu-m)>x \geq \gamma_{5}+\gamma_{6}+\gamma_{7}-m \text { and } 2 k>\varepsilon=2 m " .
\end{aligned}
$$

6. Completion of the proof. The following five lemmas conclude the proof of Theorem 1.

Lemma 17. If $H_{14}=\left\{S^{\prime \prime}: T=\gamma_{5}+\gamma_{6}+\gamma_{7}+2 m\right\}$ then $\# H_{14} \ll \mu^{5} A$.

Pr o o f. Given $M_{5}, M_{6}, M_{7}$ there are $\ll h / p^{\min (2 k+4 m, \mu+3 m)-\gamma_{5}-\gamma_{6}-\gamma_{7}-2 m}$ +1 choices for $M_{4}$ from (24) and (28). Hence

$$
\begin{aligned}
\# H_{14} \ll & h\left(\frac{h}{p^{\mu}}+1\right) \\
& \times \sum_{k, m}\left(\frac{h}{p^{\max (\mu-2 m, 2 k-m)}}+1\right)\left(\frac{h}{p^{\min (2 k+2 m, \mu+m)-\gamma_{5}-\gamma_{6}-\gamma_{7}}}+1\right) \\
& \times\left(\frac{h}{p^{\mu-k}}+1\right) \sum_{\gamma_{5}, \gamma_{6}, \gamma_{7}}\left(\frac{h}{p^{\gamma_{5}}}+1\right)\left(\frac{h}{p^{\gamma_{6}}}+1\right)\left(\frac{h}{p^{\gamma_{7}}}+1\right) .
\end{aligned}
$$

By (23) we know that $\min (2 k+2 m, \mu+m)-\gamma_{5}-\gamma_{6}-\gamma_{7} \leq \min (2 k-m, \mu-$ $2 m) \leq \max (2 k-m, \mu-2 m)$ and so the above becomes

$$
\# H_{14} \ll h\left(\frac{h}{p^{\mu}}+1\right) \sum_{\substack{k, m \\ \gamma_{5}, \gamma_{6}, \gamma_{7}}}\left(\frac{h^{6}}{p^{D_{16}}}+\frac{h^{5}}{p^{D_{17}}}+\frac{h^{4}}{p^{D_{18}}}+h^{3}\right),
$$

where

$$
\begin{aligned}
D_{16} & =2 \mu+k \geq 2 \mu+\left[\frac{\mu}{2}\right]-\left[\frac{\mu}{4}\right], \\
D_{17} & =\min \left(2 \mu+k-\gamma_{5}-\gamma_{6}-\gamma_{7}, \mu+k+2 m, 2 \mu+m-k\right) \\
& >\mu+k \geq \mu+\left[\frac{\mu}{2}\right]-\left[\frac{\mu}{4}\right],
\end{aligned}
$$




$$
\begin{aligned}
& D_{18}= \min \left(\mu-k+\gamma_{5}+\gamma_{6}+\gamma_{7},\right. \\
&\left.\min (\mu+2 k, \mu+k+2 m, 2 \mu-k+m)-\gamma_{5}-\gamma_{6}-\gamma_{7}\right) \\
&>\mu-k \geq \mu-\left[\frac{\mu}{2}\right] .
\end{aligned}
$$

LEMma 18. If $H_{15}=\left\{S^{\prime \prime}: T>\gamma_{5}+\gamma_{6}+\gamma_{7}+2 m\right.$ and $\left.U=\gamma_{5}+\gamma_{6}+\gamma_{7}+m\right\}$ then $\# H_{15} \ll \mu^{5} \mathrm{~A}$.

Pr o of. From (24) and (29) there are

$$
\ll h / p^{\left[\left(\min (2 k+4 m, \mu+3 m)-\gamma_{5}-\gamma_{6}-\gamma_{7}-m+1\right) / 2\right]}+1
$$

choices for $M_{4}$ given $M_{5}, M_{6}, M_{7}$. Therefore

$$
\begin{aligned}
\# H_{15} \ll & h\left(\frac{h}{p^{\mu}}+1\right) \\
& \times \sum_{k, m}\left(\frac{h}{p^{\left[\left(\min (2 k+3 m, \mu+2 m)-\gamma_{5}-\gamma_{6}-\gamma_{7}+1\right) / 2\right]}}+1\right) \\
& \times\left(\frac{h}{p^{\max (\mu-2 m, 2 k-m)}}+1\right)\left(\frac{h}{p^{\mu-k}}+1\right) \\
& \times \sum_{\gamma_{5}, \gamma_{6}, \gamma_{7}}\left(\frac{h}{p^{\gamma_{5}}}+1\right)\left(\frac{h}{p^{\gamma_{6}}}+1\right)\left(\frac{h}{p^{\gamma_{7}}}+1\right) .
\end{aligned}
$$

By (23) we see that

$$
\begin{aligned}
{\left[\frac{\min (2 k+3 m, \mu+2 m)-\gamma_{5}-\gamma_{6}-\gamma_{7}+1}{2}\right] } & \leq\left[\frac{\min (2 k, \mu-m)+1}{2}\right] \\
& <\max (\mu-2 m, 2 k-m)
\end{aligned}
$$

and so

$$
\# H_{15} \ll h\left(\frac{h}{p^{\mu}}+1\right) \sum_{\substack{k, m \\ \gamma_{5}, \gamma_{6}, \gamma_{7}}}\left(\frac{h^{6}}{p^{D_{19}}}+\frac{h^{5}}{p^{D_{20}}}+\frac{h^{4}}{p^{D_{21}}}+h^{3}\right),
$$

where

$$
\begin{aligned}
D_{19}= & {\left[\frac{\min (2 k+3 m, \mu+2 m)+\gamma_{5}+\gamma_{6}+\gamma_{7}+1}{2}\right] } \\
& +\max (2 \mu-2 m-k, \mu+k-m), \\
D_{20}= & {\left[\frac{\min (2 k+3 m, \mu+2 m)-\gamma_{5}-\gamma_{6}-\gamma_{7}+1}{2}\right] } \\
& +\min \left(\mu-k+\gamma_{5}+\gamma_{6}+\gamma_{7}, \max (\mu+k-m, 2 \mu-k-2 m)\right) \\
> & m+\min \left(2 \mu-k-\left[\frac{\mu}{4}\right], \mu+k-m\right) \geq \mu+\left[\frac{\mu}{2}\right]-\left[\frac{\mu}{4}\right],
\end{aligned}
$$




$$
\begin{aligned}
D_{21}=\min ( & {\left[\frac{\min (2 k+3 m, \mu+2 m)-\gamma_{5}-\gamma_{6}-\gamma_{7}+1}{2}\right] } \\
& +\min (\mu-k, \max (\mu-2 m, 2 k-m)), \\
& \left.\mu-k+\gamma_{5}+\gamma_{6}+\gamma_{7}\right)>\mu-k \geq \mu-\left[\frac{\mu}{2}\right] .
\end{aligned}
$$

From (7) and (23) it follows that if $2 k \leq \mu-m$ then

$$
\begin{aligned}
D_{19} & \geq 2 \mu+\left[\frac{\gamma_{5}+\gamma_{6}+\gamma_{7}-m+1}{2}\right] \\
& \geq 2 \mu+\left[\frac{\max (3 m, \mu-[\mu / 4])-m+1}{2}\right] \geq 2 \mu+\left[\frac{\mu}{2}\right]-\left[\frac{\mu}{4}\right]
\end{aligned}
$$

and if $2 k>\mu-m$ then

$$
\begin{aligned}
D_{19} & \geq \mu+\left[\frac{\mu-m+1}{2}\right]+\left[\frac{\mu+\max (3 m, \mu-[\mu / 4])+1}{2}\right] \\
& \geq 2 \mu+\left[\frac{\mu}{2}\right]-\left[\frac{\mu}{4}\right] .
\end{aligned}
$$

It may now be assumed that

$$
T>\gamma_{5}+\gamma_{6}+\gamma_{7}+2 m, \quad U>\gamma_{5}+\gamma_{6}+\gamma_{7}+m .
$$

From (30) it follows that $\left(2 M_{4}-M_{5}-M_{6}-M_{7}\right)\left(M_{5}^{2}+M_{6}^{2}+M_{7}^{2}-2\left(M_{5} M_{6}+\right.\right.$ $\left.\left.M_{5} M_{7}+M_{6} M_{7}\right)\right)=H p^{V}+2 M_{5} M_{6} M_{7}$ for some $H \in \mathbb{Z}$ with $p \nmid H$. Substituting this into (28) gives

$$
Q H p^{V} \equiv M_{5} M_{6} M_{7}\left(2 M_{4}-M_{5}-M_{6}-M_{7}\right)^{2}\left(\bmod p^{T}\right) .
$$

By (7) and (10) we know that $p^{\gamma_{5}+\gamma_{6}+\gamma_{7}+2 m} \|$ RHS of the above and thus, by (32), $p^{\gamma_{5}+\gamma_{6}+\gamma_{7}+2 m} \| Q H p^{V}$, from which we conclude

$$
V=\gamma_{5}+\gamma_{6}+\gamma_{7} .
$$

It can be seen from (29) that

$$
\begin{array}{r}
\left(M_{5}^{2}+M_{6}^{2}+M_{7}^{2}-2\left(M_{5} M_{6}+M_{5} M_{7}+M_{6} M_{7}\right)\right)\left(\left(2 M_{4}-M_{5}-M_{6}-M_{7}\right)^{2}+2 Q\right) \\
\equiv 6 M_{5} M_{6} M_{7}\left(2 M_{4}-M_{5}-M_{6}-M_{7}\right)\left(\bmod p^{U}\right) .
\end{array}
$$

By (7) and (10) $p^{\gamma_{5}+\gamma_{6}+\gamma_{7}+m} \|$ RHS and so, by (32), $p^{\gamma_{5}+\gamma_{6}+\gamma_{7}+m} \|$ LHS. Thus, using (10), (25) and (27), we deduce that $p^{2 m} \|\left(2 M_{4}-M_{5}-M_{6}-\right.$ $\left.M_{7}\right)^{2}+2 Q$ and

$$
x=\gamma_{5}+\gamma_{6}+\gamma_{7}-m \text {. }
$$

From (28) we have

$$
\begin{array}{r}
4 Q\left(2 M_{4}-M_{5}-M_{6}-M_{7}\right)\left(M_{5}^{2}+M_{6}^{2}+M_{7}^{2}-2\left(M_{5} M_{6}+M_{5} M_{7}+M_{6} M_{7}\right)\right) \\
-2 M_{5} M_{6} M_{7}\left(4 Q+2\left(2 M_{4}-M_{5}-M_{6}-M_{7}\right)^{2}\right) \equiv 0\left(\bmod p^{T}\right) .
\end{array}
$$


As

$4 Q=\left(2 M_{4}-M_{5}-M_{6}-M_{7}\right)^{2}-\left(M_{5}^{2}+M_{6}^{2}+M_{7}^{2}-2\left(M_{5} M_{6}+M_{5} M_{7}+M_{6} M_{7}\right)\right)$

this can be rewritten as

$$
\begin{aligned}
\left(\left(2 M_{4}-M_{5}-M_{6}-\right.\right. & \left.\left.M_{7}\right)^{3}+2 M_{5} M_{6} M_{7}\right) \\
& \times\left(M_{5}^{2}+M_{6}^{2}+M_{7}^{2}-2\left(M_{5} M_{6}+M_{5} M_{7}+M_{6} M_{7}\right)\right) \\
-\left(2 M_{4}-M_{5}-M_{6}-\right. & \left.M_{7}\right)\left(M_{5}^{2}+M_{6}^{2}+M_{7}^{2}-2\left(M_{5} M_{6}+M_{5} M_{7}+M_{6} M_{7}\right)\right) \\
& -6 M_{5} M_{6} M_{7}\left(2 M_{4}-M_{5}-M_{6}-M_{7}\right)^{2} \equiv 0\left(\bmod p^{T}\right) .
\end{aligned}
$$

It follows from (7), (10), (25) and (34) that

$$
\begin{aligned}
& \left(2 M_{4}-M_{5}-M_{6}-M_{7}\right)^{3}\left(M_{5}^{2}+M_{6}^{2}+M_{7}^{2}-2\left(M_{5} M_{6}+M_{5} M_{7}+M_{6} M_{7}\right)\right) \\
& -6 M_{5} M_{6} M_{7}\left(2 M_{4}-M_{5}-M_{6}-M_{7}\right)^{2} \equiv 0\left(\bmod p^{\min \left(T, 2\left(\gamma_{5}+\gamma_{6}+\gamma_{7}\right)-m\right)}\right),
\end{aligned}
$$

which, together with (10), implies that

$$
\begin{array}{r}
\left(2 M_{4}-M_{5}-M_{6}-M_{7}\right)\left(M_{5}^{2}+M_{6}^{2}+M_{7}^{2}-2\left(M_{5} M_{6}+M_{5} M_{7}+M_{6} M_{7}\right)\right) \\
-6 M_{5} M_{6} M_{7} \equiv 0\left(\bmod p^{\min \left(T-2 m, 2\left(\gamma_{5}+\gamma_{6}+\gamma_{7}\right)-3 m\right)}\right) .
\end{array}
$$

By (29),

$$
\begin{array}{r}
\left(2\left(2 M_{4}-M_{5}-M_{6}-M_{7}\right)^{2}+4 Q\right)\left(M_{5}^{2}+M_{6}^{2}+M_{7}^{2}-2\left(M_{5} M_{6}+M_{5} M_{7}+M_{6} M_{7}\right)\right) \\
-12 M_{5} M_{6} M_{7}\left(2 M_{4}-M_{5}-M_{6}-M_{7}\right) \equiv 0\left(\bmod p^{U}\right) .
\end{array}
$$

Substituting for $4 Q$ this becomes

$$
\begin{array}{r}
3\left(2 M_{4}-M_{5}-M_{6}-M_{7}\right)^{2}\left(M_{5}^{2}+M_{6}^{2}+M_{7}^{2}-2\left(M_{5} M_{6}+M_{5} M_{7}+M_{6} M_{7}\right)\right) \\
-\left(M_{5}^{2}+M_{6}^{2}+M_{7}^{2}-2\left(M_{5} M_{6}+M_{5} M_{7}+M_{6} M_{7}\right)\right)^{2} \\
-12 M_{5} M_{6} M_{7}\left(2 M_{4}-M_{5}-M_{6}-M_{7}\right) \equiv 0\left(\bmod p^{U}\right),
\end{array}
$$

which, by (10), (25) and (34), reduces to

$$
\begin{array}{r}
\left(2 M_{4}-M_{5}-M_{6}-M_{7}\right)\left(M_{5}^{2}+M_{6}^{2}+M_{7}^{2}-2\left(M_{5} M_{6}+M_{5} M_{7}+M_{6} M_{7}\right)\right) \\
-4 M_{5} M_{6} M_{7} \equiv 0\left(\bmod p^{\min \left(U-m, 2\left(\gamma_{5}+\gamma_{6}+\gamma_{7}\right)-3 m\right)}\right) .
\end{array}
$$

Subtracting (35) from the above congruence we obtain $2 M_{5} M_{6} M_{7} \equiv 0$ $\left(\bmod p^{\min \left(U-m, T-2 m, 2\left(\gamma_{5}+\gamma_{6}+\gamma_{7}\right)-3 m\right)}\right)$ and so, by $(7), \gamma_{5}+\gamma_{6}+\gamma_{7} \geq \min (U-$ $\left.m, T-2 m, 2\left(\gamma_{5}+\gamma_{6}+\gamma_{7}\right)-3 m\right)$. This, together with (7), (23) and (32), implies that

$$
\mu-\left[\frac{\mu}{4}\right] \leq 3 m=\gamma_{5}+\gamma_{6}+\gamma_{7}<\mu .
$$

It follows from (36) that

$$
\left[\frac{\mu}{2}\right]-\left[\frac{\mu}{4}\right] \leq m<k \leq\left[\frac{\mu}{2}\right] \leq 2 m
$$


LEMMA 19. If $H_{16}=\left\{S^{\prime \prime}:\right.$ (32) holds and $\left.2 U \geq \min (\mu+5 m, 2 k+6 m)\right\}$ then $\# H_{16} \ll \mu^{5} A$.

Proof. Given $M_{5}, M_{6}, M_{7}$ there are $\ll h / p^{U-3 m}+1$ choices for $M_{4}$ from (29), (30), (33) and (36). As

$$
U \geq \min \left(\left[\frac{\mu+5 m+1}{2}\right], k+3 m\right)
$$

we may take the number of $M_{4}$ to be $\ll h / p^{\min (k,[(\mu-m+1) / 2])}+1$. Using (36) it follows that

$$
\begin{aligned}
\# H_{16} \ll & h\left(\frac{h}{p^{\mu}}+1\right) \\
& \times \sum_{k, m}\left(\frac{h}{p^{\min ([(\mu-m+1) / 2], k)}}+1\right)\left(\frac{h}{p^{\max (\mu-2 m, 2 k-m)}}+1\right) \\
& \times\left(\frac{h}{p^{\mu-k}}+1\right) \sum_{\gamma_{5}, \gamma_{6}, \gamma_{7}}\left(\frac{h}{p^{\gamma_{5}}}+1\right)\left(\frac{h}{p^{\gamma_{6}}}+1\right)\left(\frac{h}{p^{\gamma_{7}}}+1\right) \\
\ll & \mu^{3} h\left(\frac{h}{p^{\mu}}+1\right) \sum_{k, m}\left(\frac{h^{6}}{p^{D_{22}}}+\frac{h^{5}}{p^{D_{23}}}+\frac{h^{4}}{p^{D_{24}}}+h^{3}\right),
\end{aligned}
$$

where

$$
\begin{aligned}
D_{22}= & \mu-k+3 m+\min \left(k,\left[\frac{\mu-m+1}{2}\right]\right)+\max (\mu-2 m, 2 k-m) \\
\geq & 2 \mu+m \geq 2 \mu+\left[\frac{\mu}{2}\right]-\left[\frac{\mu}{4}\right], \\
D_{23}= & \min \left(k,\left[\frac{\mu-m+1}{2}\right]\right) \\
& +\min (\mu-k+3 m, \max (2 \mu-k-2 m, \mu+k-m)) \\
> & \mu+\min \left(k,\left[\frac{\mu-m+1}{2}\right]\right)>\mu+\left[\frac{\mu}{2}\right]-\left[\frac{\mu}{4}\right], \\
D_{24}= & \min \left(\mu-k+3 m, \min \left(k,\left[\frac{\mu-m+1}{2}\right]\right)\right. \\
& +\min (\mu-k, \max (2 k-m, \mu-2 m))) \\
> & \min (\mu-k, 2 k) \geq \mu-\left[\frac{\mu}{2}\right] .
\end{aligned}
$$


For the final two cases it may be assumed that

$$
2 U<\min (2 k+6 m, \mu+5 m) .
$$

We rewrite (24) as

(38) $A_{4} M_{4}^{4}+A_{3} M_{4}^{3}+A_{2} M_{4}^{2}+A_{1} M_{4}+A_{0} \equiv 0\left(\bmod p^{\min (2 k+4 m, \mu+3 m)}\right)$.

Hence (28) and (29) now become

$$
p^{T} \|\left(4 A_{4} M_{4}^{3}+3 A_{3} M_{4}^{2}+2 A_{2} M_{4}+A_{1}, p^{\min (2 k+4 m, \mu+3 m)}\right)
$$

and

$$
p^{U} \|\left(12 A_{4} M_{4}^{2}+6 A_{3} M_{4}+2 A_{2}, p^{\min (2 k+4 m, \mu+3 m)}\right)
$$

where

$$
\begin{aligned}
& A_{4}=\sigma_{1}^{2}-4 \sigma_{2}=M_{5}^{2}+M_{6}^{2}+M_{7}^{2}-2\left(M_{5} M_{6}+M_{5} M_{7}+M_{6} M_{7}\right) \\
& A_{3}=8 \sigma_{1} \sigma_{2}-2 \sigma_{1}^{3}-4 M_{5} M_{6} M_{7} \\
& A_{2}=\sigma_{1}^{4}-2 \sigma_{1}^{2} \sigma_{2}-8 \sigma_{2}^{2}+6 M_{5} M_{6} M_{7} \sigma_{1} \\
& A_{1}=8 \sigma_{1} \sigma_{2}^{2}-2 \sigma_{1}^{3} \sigma_{2}-4 M_{5} M_{6} M_{7} \sigma_{2}-2 M_{5} M_{6} M_{7} \sigma_{1}^{2} \\
& A_{0}=\sigma_{1}^{2} \sigma_{2}^{2}-4 \sigma_{2}^{3}+2 M_{5} M_{6} M_{7} \sigma_{1} \sigma_{2}+M_{5}^{2} M_{6}^{2} M_{7}^{2}
\end{aligned}
$$

and

$$
\sigma_{1}=M_{5}+M_{6}+M_{7} \quad \text { and } \quad \sigma_{2}=M_{5} M_{6}+M_{5} M_{7}+M_{6} M_{7} .
$$

From (39) we see that for some $R \in \mathbb{Z}, p \nmid R$,

$$
M_{4}^{3}=\frac{R p^{T}-3 A_{3} M_{4}^{2}-2 A_{2} M_{4}-A_{1}}{4 A_{4}} .
$$

This, in conjunction with (38), implies that

$$
\begin{aligned}
A_{0}-\frac{A_{1} A_{3}}{16 A_{4}}+\frac{A_{3} R p^{T}}{16 A_{4}}+M_{4}\left(\frac{R p^{T}}{4}+\frac{3 A_{1}}{4}-\right. & \left.\frac{A_{2} A_{3}}{8 A_{4}}\right)+M_{4}^{2}\left(\frac{A_{2}}{2}-\frac{3 A_{3}^{2}}{16 A_{4}}\right) \\
& \equiv 0\left(\bmod p^{\min (2 k+4 m, \mu+3 m)}\right) .
\end{aligned}
$$

But from (25), (34) and (36) we know that $p^{2 m} \| A_{4}$ and thus

$$
\begin{array}{r}
16 A_{0} A_{4}-A_{1} A_{3}+A_{3} R p^{T}+M_{4}\left(4 A_{4} R p^{T}+12 A_{1} A_{4}-2 A_{2} A_{3}\right) \\
+M_{4}^{2}\left(8 A_{2} A_{4}-3 A_{3}^{2}\right) \equiv 0\left(\bmod p^{\min (2 k+6 m, \mu+5 m)}\right) .
\end{array}
$$

Clearly from (40), $p^{U} \| 6 A_{4} M_{4}^{2}+3 A_{3} M_{4}+A_{2}$ and so $36 A_{4}^{2} M_{4}^{4}+36 A_{3} A_{4} M_{4}^{3}+M_{4}^{2}\left(9 A_{3}^{2}+12 A_{2} A_{4}\right)+6 A_{2} A_{3} M_{4}+A_{2}^{2} \equiv 0\left(\bmod p^{2 U}\right)$.

Also, by (38),

$36 A_{4}\left(A_{4} M_{4}^{4}+A_{3} M_{4}^{3}+A_{2} M_{4}^{2}+A_{1} M_{4}+A_{0}\right) \equiv 0\left(\bmod p^{\min (2 k+6 m, \mu+5 m)}\right)$.

These two congruences and (37) imply that

$M_{4}^{2}\left(24 A_{2} A_{4}-9 A_{3}^{2}\right)+M_{4}\left(36 A_{1} A_{4}-6 A_{2} A_{3}\right)+36 A_{0} A_{4}-A_{2}^{2} \equiv 0\left(\bmod p^{2 U}\right)$. 
Substituting this into (42) produces

$$
12 A_{0} A_{4}-3 A_{1} A_{3}+A_{2}^{2}+3 R p^{T}\left(A_{3}+4 A_{4} M_{4}\right) \equiv 0\left(\bmod p^{2 U}\right) .
$$

From (41) we know that

$$
A_{3}+4 A_{4} M_{4}=2 A_{4}\left(2 M_{4}-M_{5}-M_{6}-M_{7}\right)-4 M_{5} M_{6} M_{7} .
$$

Thus

$$
\begin{array}{r}
12 A_{0} A_{4}-3 A_{1} A_{3}+A_{2}^{2}+6 R p^{T}\left(A_{4}\left(2 M_{4}-M_{5}-M_{6}-M_{7}\right)-2 M_{5} M_{6} M_{7}\right) \\
\equiv 0\left(\bmod p^{2 U}\right)
\end{array}
$$

which, taken with (7), (10) and (36), implies that $p^{\min (T+3 m, 2 U)} \mid 12 A_{0} A_{4}$ $3 A_{1} A_{3}+A_{2}^{2}$. By (41) we can rewrite this as

$$
A_{4}^{4}+24 M_{5}^{2} M_{6}^{2} M_{7}^{2} A_{4} \equiv 0\left(\bmod p^{\min (T+3 m, 2 U)}\right),
$$

or

$$
A_{4}^{3}+24 M_{5}^{2} M_{6}^{2} M_{7}^{2} \equiv 0\left(\bmod p^{\min (T+m, 2 U-2 m)}\right) .
$$

It is now necessary to examine the derivatives of $A_{4}^{3}+24 M_{5}^{2} M_{6}^{2} M_{7}^{2}$ with respect to $M_{7}$. Define

$$
\begin{aligned}
& p^{\delta_{1}} \| 3 A_{4}^{2}\left(2 M_{7}-2 M_{5}-2 M_{6}\right)+48 M_{5}^{2} M_{6}^{2} M_{7}, \\
& p^{\delta_{2}} \| 6 A_{4}^{2}+6 A_{4}\left(2 M_{7}-2 M_{5}-2 M_{6}\right)^{2}+48 M_{5}^{2} M_{6}^{2}, \\
& p^{\delta_{3}} \| 36 A_{4}\left(2 M_{7}-2 M_{5}-2 M_{6}\right)+6\left(2 M_{7}-2 M_{5}-2 M_{6}\right)^{3}, \\
& p^{\delta_{4}} \| 72 A_{4}+72\left(2 M_{7}-2 M_{5}-2 M_{6}\right)^{2}, \\
& p^{\delta_{5}} \| 720\left(M_{7}-M_{5}-M_{6}\right) .
\end{aligned}
$$

By assumption $\gamma_{5} \geq \gamma_{6} \geq \gamma_{7}$ and so from (36) we have

$$
\gamma_{5}+\gamma_{6} \geq 2 m \text {. }
$$

Therefore, as $p^{2 m} \| A_{4}=\left(M_{7}-M_{5}-M_{6}\right)^{2}-4 M_{5} M_{6}$, it follows that $p^{m} \mid M_{7}-M_{5}-M_{6}$ and from (44) we deduce that

$$
\delta_{5} \geq m, \quad \delta_{4} \geq 2 m, \quad \delta_{3} \geq 3 m .
$$

Lemma 20. If $H_{17}=\left\{S^{\prime \prime}:(32),(37)-(41)\right.$ and (44) hold and $\left.\delta_{4}=2 m\right\}$ then $\# H_{17} \ll \mu^{6} A$.

Proof. The proof is split into two cases according to the value of $\min (2 U-2 m, T+m)$.

Case 1: $2 U-2 m \leq T+m$. Given $M_{5}, M_{6}, M_{7}$ there are

$$
\ll h / p^{\max (\min (\mu+3 m, 2 k+4 m)-T, T-U)}+1
$$

choices for $M_{4}$ from (38), (39) and (40). Since

$\max (\min (\mu+3 m, 2 k+4 m)-T, T-U) \geq\left[\frac{\min (\mu+3 m, 2 k+4 m)-U+1}{2}\right]$ 
we may take the number of $M_{4}$ to be $\ll h / p^{[(\min (\mu+3 m, 2 k+4 m)-U+1) / 2]}+1$. Given $M_{5}, M_{6}$ there are $\ll h / p^{W}+1$ choices for $M_{7}$ from (43) and (44), where

$$
W=\max \left(2 U-2 m-\delta_{1}, \delta_{1}-\delta_{2}, \delta_{2}-\delta_{3}, \delta_{3}-2 m\right) \geq\left[\frac{U+1}{2}\right]-m .
$$

Thus

$$
\begin{aligned}
\# H_{17} & \ll h\left(\frac{h}{p^{\mu}}+1\right) \\
& \times \sum_{k, m, U}\left(\frac{h}{p^{[(\min (\mu+3 m, 2 k+4 m)-U+1) / 2]}}+1\right)\left(\frac{h}{p^{[(U+1) / 2]-m}}+1\right) \\
& \times\left(\frac{h}{p^{\mu-k}}+1\right)\left(\frac{h}{p^{\max (\mu-2 m, 2 k-m)}}+1\right) \sum_{\gamma_{5}, \gamma_{6}}\left(\frac{h}{p^{\gamma_{5}}}+1\right)\left(\frac{h}{p^{\gamma_{6}}}+1\right) .
\end{aligned}
$$

By (37),

$$
\left[\frac{\min (\mu+3 m, 2 k+4 m)-U+1}{2}\right] \geq\left[\frac{U+1}{2}\right]-m
$$

and so, using (45), we obtain

$$
\begin{aligned}
\# H_{17} \ll & \mu^{2} h\left(\frac{h}{p^{\mu}}+1\right) \\
& \times \sum_{k, m, U}\left(\frac{h^{2}}{p^{\min ([(\mu+m+1) / 2], k+m)}}+\frac{h}{p^{[(U+1) / 2]-m}}+1\right) \\
& \times\left(\frac{h^{4}}{p^{\max (2 \mu-k, \mu+k+m)}}+\frac{h^{3}}{p^{\mu}}+\frac{h^{2}}{p^{k}}+h\right) \\
\ll & \mu^{2} h\left(\frac{h}{p^{\mu}}+1\right) \sum_{k, m, U}\left(\frac{h^{6}}{p^{D_{25}}}+\frac{h^{5}}{p^{D_{26}}}+\frac{h^{4}}{p^{D_{27}}}+h^{3}\right),
\end{aligned}
$$

where

$$
\begin{aligned}
& D_{25}= \min \left(\left[\frac{\mu+m+1}{2}\right], k+m\right)+\max (2 \mu-k, \mu+k+m) \\
& \geq 2 \mu+m \geq 2 \mu+\left[\frac{\mu}{2}\right]-\left[\frac{\mu}{4}\right], \\
& D_{26}=\min \left(\mu+\min \left(\left[\frac{\mu+m+1}{2}\right], k+m\right),\right. \\
& \\
&\left.\quad\left[\frac{U+1}{2}\right]-m+\max (2 \mu-k, \mu+k+m)\right) \\
&>\mu+k>\mu+\left[\frac{\mu}{2}\right]-\left[\frac{\mu}{4}\right],
\end{aligned}
$$




$$
\begin{gathered}
D_{27}=\min \left(\mu+\left[\frac{U+1}{2}\right]-m, k+\min \left(\left[\frac{\mu+m+1}{2}\right], k+m\right),\right. \\
\max (2 \mu-k, \mu+k+m))>\mu-\left[\frac{\mu}{2}\right] .
\end{gathered}
$$

Case $2: T+M<2 U-2 m$. There are

$$
\ll h / p^{\max (\min (\mu+3 m, 2 k+4 m)-T, U-3 m)}+1
$$

choices for $M_{4}$ from (29), (30), (33), (36), (38) and (39), given $M_{5}, M_{6}, M_{7}$. Since

$$
\begin{aligned}
\max (\min (\mu+3 m, 2 k+4 m)-T, U-3 m) & \\
\geq & \left.\geq \frac{\min (\mu+3 m, 2 k+4 m)-T+U-3 m+1}{2}\right]
\end{aligned}
$$

we may take the number of $M_{4}$ to be $\ll h / p^{[(\min (\mu, 2 k+m)-T+U+1) / 2]}+1$. Given $M_{5}, M_{6}$, there are $\ll h / p^{Y}+1$ choices for $M_{7}$ from (43) and (44), where $Y=\max \left(T+m-\delta_{1}, \delta_{1}-\delta_{2}, \delta_{2}-\delta_{3}, \delta_{3}-2 m\right) \geq[(T-m+3) / 4]$ and so

$$
\begin{aligned}
\# H_{17} \ll & h\left(\frac{h}{p^{\mu}}+1\right) \\
& \times \sum_{\substack{k, m \\
U, T}}\left(\frac{h}{p^{[(\min (\mu, 2 k+m)-T+U+1) / 2]}}+1\right)\left(\frac{h}{p^{[(T-m+3) / 4]}}+1\right) \\
& \times\left(\frac{h}{p^{\mu-k}}+1\right)\left(\frac{h}{p^{\max (\mu-2 m, 2 k-m)}}+1\right) \\
& \times \sum_{\gamma_{5}, \gamma_{6}}\left(\frac{h}{p^{\gamma_{5}}}+1\right)\left(\frac{h}{p^{\gamma_{6}}}+1\right) .
\end{aligned}
$$

From (37),

$$
\begin{aligned}
{\left[\frac{\min (\mu, 2 k+m)-T+U+1}{2}\right] } & +\left[\frac{T-m+3}{4}\right] \\
& \geq\left[\frac{\min (2 \mu-m, 4 k+m)+2 U-T}{4}\right] \\
& \geq \min \left(k+m,\left[\frac{\mu+m}{2}\right]\right)
\end{aligned}
$$

and

$$
\left[\frac{\min (\mu, 2 k+m)-T+U+1}{2}\right] \geq \min \left(\left[\frac{k+m}{2}\right],\left[\frac{\mu+m}{4}\right]\right) .
$$


Also, (32) and (36) imply that $T>5 m$ and consequently $[(T-m+3) / 4]>m$. Hence, by (45), it follows that

$$
\begin{aligned}
\# H_{17} \ll & \mu^{4} h\left(\frac{h}{p^{\mu}}+1\right) \sum_{k, m}\left(\frac{h^{2}}{p^{\min ([(\mu+m) / 2], k+m)}}+\frac{h}{p^{m}}+1\right) \\
& \times\left(\frac{h^{4}}{p^{\max (2 \mu-k, \mu+k+m)}}+\frac{h^{3}}{p^{\mu}}+\frac{h^{2}}{p^{k}}+h\right) \\
\ll & \mu^{4} h\left(\frac{h}{p^{\mu}}+1\right) \sum_{k, m}\left(\frac{h^{6}}{p^{D_{28}}}+\frac{h^{5}}{p^{D_{29}}}+\frac{h^{4}}{p^{D_{30}}}+h^{3}\right),
\end{aligned}
$$

where

$$
\begin{aligned}
& D_{28}= \min \left(\left[\frac{\mu+m}{2}\right], k+m\right)+\max (2 \mu-k, \mu+k+m) \\
& \geq 2 \mu+m \geq 2 \mu+\left[\frac{\mu}{2}\right]-\left[\frac{\mu}{4}\right], \\
& D_{29}=\min \left(\mu+\min \left(\left[\frac{\mu+m}{2}\right], k+m\right),\right. \\
&\left.>\mu+k>\mu+\left[\frac{\mu}{2}\right]-\left[\frac{\mu}{4}\right], \quad \max (2 \mu-k+m, \mu+k+2 m)\right) \\
& D_{30}=\min \left(\mu+m, k+\min \left(\left[\frac{\mu+m}{2}\right], k+m\right),\right. \\
&\quad \max (2 \mu-k, \mu+k+m))>\mu-\left[\frac{\mu}{2}\right] .
\end{aligned}
$$

Lemma 21. If $H_{18}=\left\{S^{\prime \prime}:(32),(37)-(41)\right.$ and (44) hold and $\left.\delta_{4} \neq 2 m\right\}$ then $\# H_{18} \ll \mu^{6} A$.

Proof. By (46) we must have $\delta_{4}>2 m$. Using (44) it follows that $A_{4}+$ $\left(2 M_{7}-2 M_{5}-2 M_{6}\right)^{2} \equiv 0\left(\bmod p^{\delta_{4}}\right)$ and so we deduce that

$$
p^{m} \| M_{7}-M_{5}-M_{6} \text {. }
$$

This together with (44) implies that $\delta_{5}=m$ and

$$
6 A_{4}+\left(2 M_{7}-2 M_{5}-2 M_{6}\right)^{2} \equiv 0\left(\bmod p^{\delta_{3}-m}\right) .
$$

It can also be seen from (44) that

$$
6 A_{4}+6\left(2 M_{7}-2 M_{5}-2 M_{6}\right)^{2} \equiv 0\left(\bmod p^{\delta_{4}}\right) .
$$


Combining these two congruences gives

$$
5\left(2 M_{7}-2 M_{5}-2 M_{6}\right)^{2} \equiv 0\left(\bmod p^{\min \left(\delta_{3}-m, \delta_{4}\right)}\right),
$$

which, by (47), implies that $2 m \geq \min \left(\delta_{3}-m, \delta_{4}\right)$ and thus $\delta_{3}=3 m$.

Case 1: $2 U-2 m \leq T+m$. Given $M_{5}, M_{6}, M_{7}$ there are

$$
\ll h / p^{[(\min (\mu+3 m, 2 k+4 m)-U+1) / 2]}+1
$$

choices for $M_{4}$ as in Lemma 20, Case 1. From (43) and (44) we have « $h / p^{Z}+1$ choices for $M_{7}$, given $M_{5}, M_{6}$, where

$$
Z=\max \left(2 U-2 m-\delta_{1}, \delta_{1}-\delta_{2}, \delta_{2}-3 m\right) \geq\left[\frac{2 U-5 m+2}{3}\right] .
$$

Therefore

$$
\begin{aligned}
\# H_{18} \ll & h\left(\frac{h}{p^{\mu}}+1\right) \\
& \times \sum_{k, m, U}\left(\frac{h}{p^{[(\min (\mu+3 m, 2 k+4 m)-U+1) / 2]}}+1\right)\left(\frac{h}{p^{[(2 U-5 m+2) / 3]}}+1\right) \\
& \times\left(\frac{h}{p^{\mu-k}}+1\right)\left(\frac{h}{p^{\max (\mu-2 m, 2 k-m)}}+1\right) \sum_{\gamma_{5}, \gamma_{6}}\left(\frac{h}{p^{\gamma_{5}}}+1\right)\left(\frac{h}{p^{\gamma_{6}}}+1\right) .
\end{aligned}
$$

It can be seen from $(32)$ and $(36)$ that $U>4 m$, which in turn implies that $[(2 U-5 m+2) / 3]>m$ and

$$
\begin{aligned}
{\left[\frac{\min (\mu+3 m, 2 k+4 m)-U+1}{2}\right]+} & {\left[\frac{2 U-5 m+2}{3}\right] } \\
& \geq\left[\frac{\min (3 \mu-m, 6 k+2 m)+U}{6}\right] \\
& \geq \min \left(\left[\frac{\mu+m}{2}\right], k+m\right) .
\end{aligned}
$$

Also,

$$
\left[\frac{\min (\mu+3 m, 2 k+4 m)-U+1}{2}\right] \geq \min \left(\left[\frac{k+m}{2}\right],\left[\frac{\mu+m}{4}\right]\right)
$$

by (37). Using (45) it follows that

$$
\begin{aligned}
\# H_{18} & \ll \mu^{3} h\left(\frac{h}{p^{\mu}}+1\right) \sum_{k, m}\left(\frac{h^{2}}{p^{\min ([(\mu+m) / 2], k+m)}}+\frac{h}{p^{m}}+1\right) \\
& \times\left(\frac{h^{4}}{p^{\max (2 \mu-k, \mu+k+m)}}+\frac{h^{3}}{p^{\mu}}+\frac{h^{2}}{p^{k}}+h\right) \\
& \ll \mu^{5} A
\end{aligned}
$$


by comparison with Case 2 of the previous lemma.

Case $2: T+m<2 U-2 m$. Given $M_{5}, M_{6}, M_{7}$ we have

$$
\ll h / p^{[(\min (\mu, 2 k+m)-T+U+1) / 2]}+1
$$

choices for $M_{4}$ as in Case 2 of Lemma 20. By (43) and (44) we have $\ll h / p^{L}+1$ choices for $M_{7}$ given $M_{5}, M_{6}$, where

$$
L=\max \left(T+m-\delta_{1}, \delta_{1}-\delta_{2}, \delta_{2}-3 m\right) \geq\left[\frac{T-2 m+2}{3}\right]
$$

and so

$$
\begin{aligned}
\# H_{18} \ll & h\left(\frac{h}{p^{\mu}}+1\right) \\
& \times \sum_{k, m}\left(\frac{h}{p^{[(\min (\mu, 2 k+m)-T+U+1) / 2]}}+1\right)\left(\frac{h}{p^{[(T-2 m+2) / 3]}}+1\right) \\
& \times\left(\frac{h}{p^{\mu-k}}+1\right)\left(\frac{h}{p^{\max (\mu-2 m, 2 k-m)}}+1\right) \sum_{\gamma_{5}, \gamma_{6}}\left(\frac{h}{p^{\gamma_{5}}}+1\right)\left(\frac{h}{p^{\gamma_{6}}}+1\right) .
\end{aligned}
$$

By (32) and (36), $T>5 m$ and $U>4 m$. Consequently, $[(T-2 m+2) / 3]>m$ and

$$
\begin{aligned}
& {\left[\frac{T-2 m+2}{3}\right]+\left[\frac{\min (\mu, 2 k+m)-T+U+1}{2}\right]} \\
& \quad \geq\left[\frac{\min (3 \mu-m, 6 k+2 m)+U}{6}\right] \geq \min \left(\left[\frac{\mu+m}{2}\right], k+m\right) .
\end{aligned}
$$

As in Lemma 20, Case 2 we have

$$
\begin{aligned}
\# H_{18} \ll & \mu^{4} h\left(\frac{h}{p^{\mu}}+1\right) \sum_{k, m}\left(\frac{h^{2}}{p^{\min ([(\mu+m) / 2], k+m)}}+\frac{h}{p^{m}}+1\right) \\
& \times\left(\frac{h^{4}}{p^{\max (2 \mu-k, \mu+k+m)}}+\frac{h^{3}}{p^{\mu}}+\frac{h^{2}}{p^{k}}+h\right) \\
& \ll \mu^{6} A .
\end{aligned}
$$

Since $\# H \ll \#\left\{\bigcup_{j=1}^{18} H_{j}\right\}$, Theorem 1 follows immediately from Lemmas 4 to 21 .

\section{References}

[1] D. A. Burgess, Estimation of character sums modulo a power of a prime, Proc. London Math. Soc. (3) 52 (1986), 215-235.

[2] -, On character sums and L-series, ibid. 12 (1962), 193-206. 
[3] D. A. Burgess, On a set of congruences related to character sums III, J. London Math. Soc. (2) 45 (1992), 201-214.

[4] L. K. Hua and S. H. Min, An analogue of Tarry's problem, Acad. Sinica Science Record 1 (1942), 26-29.

DEPARTMENT OF MATHEMATICS

NOTTINGHAM UNIVERSITY

UNIVERSITY PARK

NOTTINGHAM NG7 2RD

ENGLAND 